\title{
Quasisymmetry, measure and a question of Heinonen
}

Stephen Semmes

Abstract. In this paper we resolve in the affirmative a question of Heinonen on the absolute continuity of quasisymmetric mappings defined on subsets of Euclidean spaces. The main ingredients in the proof are extension results for quasisymmetric mappings and metric doubling measures.

\section{Introduction.}

If $F$ is a subset of $\mathbb{R}^{n}$ and $g: F \rightarrow \mathbb{R}^{n}$ is a mapping, then we say that $g$ is quasisymmetric if it is not constant and if there exists a homeomorphism $\eta:[0, \infty) \rightarrow[0, \infty)$ such that

$$
|x-y| \leq t|x-z| \quad \text { implies } \quad|g(x)-g(y)| \leq \eta(t)|g(x)-g(z)|
$$

whenever $x, y, z \in F$. We shall sometimes say that $g$ is $\eta$-quasisymmetric to be explicit, or we shall refer to $\eta$ as the function that governs the quasisymmetry of $g$ when we want to be specific but not explicit.

This condition is a little bit hard to digest at first, but it means that the mapping approximately preserves relative distances, even if it may distort distances in an unbounded manner. In other words, if $x$ is a lot closer to $y$ than to $z$, then the corresponding property for $g(x), g(y)$, and $g(z)$ should also hold, even though the distances themselves may change dramatically. For instance, the mapping defined by $g(x)=a x$ 
is $\eta$-quasisymmetric with $\eta(t) \equiv t$ for all positive numbers $a$, but this mapping distorts distances strongly when $a$ is very large or very small.

See [TV] for basic facts about quasisymmetric mappings.

In the case of mappings defined on all of $\mathbb{R}^{n}$ the quasisymmetry condition is equivalent to the more famous quasiconformal condition, which is an infinitesimal version of the same idea. It turns out that quasisymmetric mappings on $\mathbb{R}^{n}$ send sets of measure zero to sets of measure zero when $n>1$, see [V1]. This is not true when $n=1$, because of an example in [BA].

Problem 1.2. (Juha Heinonen.) If $F$ is a compact subset of $\mathbb{R}^{n}, n>1$, and $g: F \rightarrow \mathbb{R}^{n}$ is quasisymmetric, is it true that $g(F)$ has Lebesgue measure zero if $F$ has Lebesgue measure zero?

We shall see that the answer is yes. The proof will not give a new approach to the result for global quasisymmetric mappings, instead it will work by reducing to a method of Gehring [G] for the global case. Note however that quasisymmetric maps defined on subsets of $\mathbb{R}^{n}$ need not extend to global quasisymmetric mappings, so that the most obvious path to reducing to the global case is not available to us.

It will be more convenient to use the following reformulation of this problem.

Theorem 1.3. Let $F$ be a compact subset of $\mathbb{R}^{n}, n>1$, and suppose that $g: F \rightarrow \mathbb{R}^{n}$ is quasisymmetric. Then $g(F)$ has positive Lebesgue measure if $F$ has positive Lebesgue measure.

Let us check that this resolves Problem 1.2.

Lemma 1.4. If $g: F \rightarrow \mathbb{R}^{n}$ is quasisymmetric, then $g^{-1}: g(F) \rightarrow \mathbb{R}^{n}$ makes sense and is quasisymmetric.

This is well-known and easy, but let us go quickly through the proof for the sake of completeness. Our mapping $g$ is injective if it is quasisymmetric, so that its inverse is well-defined. From (1.1) we have that

(1.5) $\eta(t)|g(x)-g(z)|<|g(x)-g(y)| \quad$ implies $\quad t|x-z|<|x-y|$.

One can sort this out to see that $g^{-1}$ is quasisymmetric, but with $\eta(t)$ replaced by $\left(\eta^{-1}(1 / t)\right)^{-1}$. This proves the lemma. 
To see that Theorem 1.3 implies a positive answer to Problem 1.2 one need only switch from $g$ to $g^{-1}$ using the lemma.

In order to prove Theorem 1.3 we shall make some modifications to $g$ and $F$. It would be simpler if we could just extend $g$ to a mapping on all of $\mathbb{R}^{n}$, but this is not possible in general. Our plan will be to replace $g$ with a map which lives on a thick set, and then to show that the pull-back of Lebesgue measure under this mapping behaves well.

The modifications of $g$ will proceed in steps. Basically we want to progressively thicken the domain $F$ of $g$. We begin with a definition.

Definition 1.6. Let $F_{0}, F$ be subsets of $\mathbb{R}^{n}$, with $F_{0} \subseteq F$. We shall say that $F_{0}$ is a serious subset of $F$ if there exists a constant $C>0$ so that if $x \in F_{0}$ and $0<t<\operatorname{diam} F_{0}$, then there is a point $y \in F$ such that

$$
C^{-1} t \leq|x-y| \leq t
$$

We say that $F$ is serious if it is serious as a subset of itself.

This is a mild nondegeneracy condition which forbids isolated islands in a quantitative and uniform way. This is useful for the quasisymmetry condition (1.1), which provides information only about relative distances.

The property of a set being serious has been considered before under various names (unknown to the author until it was too late) such as "uniformly perfect" and "homogeneously dense", and it is a special case of the thickness conditions discussed in [VVW]. It may be that the relative property for subsets was not considered before.

We are going to be working with serious sets, and it would be nice if we could find a serious set of positive measure inside any given set of positive measure. Unfortunately this turns out not to be true, Pertti Mattila tells me that there are counterexamples. The following simple observation will suffice for our purposes.

Lemma 1.8. Let $F$ be a compact subset of $\mathbb{R}^{n}$ with positive measure. Then for each $\varepsilon>0$ there is a compact subset $F_{0}$ of $F$ such that $F_{0}$ is a serious subset of $F$ and $\left|F_{0}\right|>|F|-\varepsilon$.

We do not give bounds on the seriousness constant here.

To prove this we use points of density and Egoroff's theorem. From Lebesgue's theorem we know that almost every element of $F$ is a point 
of density of $F$. That is,

$$
\lim _{j \rightarrow \infty} \frac{\left|F \cap B\left(x, 2^{-j}\right)\right|}{\left|B\left(x, 2^{-j}\right)\right|}=1
$$

for almost all $x \in F$. Let $\varepsilon>0$ be given. By Egoroff's theorem we can find a measurable subset $F_{0}$ of $F$ on which we have uniform convergence for this limit and $\left|F_{0}\right|>|F|-\varepsilon$. We can take $F_{0}$ to be compact because we can always replace it, if necessary, with a compact subset with almost the same measure.

Uniform convergence implies that there is a $\delta>0$ such that

$$
\frac{\left|F \cap B\left(x, 2^{-j}\right)\right|}{\left|B\left(x, 2^{-j}\right)\right|} \geq \frac{1}{2}
$$

when $x \in F_{0}$ and $0<2^{-j}<\delta$. It is not hard to see that this implies that $F_{0}$ is a serious subset of $F$, but with a horrible constant which depends on $\delta$. (At scales finer than $\delta$ the constant is bounded. In other words, we could control the seriousness constant if we were willing to give up control on the measure.) This proves Lemma 1.8.

Of course (1.9) is much stronger than seriousness, but seriousness is a more natural condition for most of what we shall do.

Given a quasisymmetric mapping defined on some set we would like to modify it to get a mapping which is defined on a thicker set. The next result will be the first step of such a process, and then we shall go another step afterwards.

Proposition 1.10. Suppose that $F$ is a closed subset of $\mathbb{R}^{n}$, that $g$ : $F \rightarrow \mathbb{R}^{n}$ is quasisymmetric, and that $F_{0}$ is a closed serious subset of $F$. Then we can find a serious closed set $F^{*}$ in $\mathbb{R}^{n}$ which contains $F_{0}$ (but need not be contained in $F$ ) and a quasisymmetric mapping $g^{*}: F^{*} \rightarrow \mathbb{R}^{n}$ such that $g^{*}=g$ on $F_{0}$. The seriousness constant for $F^{*}$ and the function $\eta^{*}$ which controls the quasisymmetry of $g^{*}$ are controlled in terms of the dimension, the seriousness constant for $\left(F_{0}, F\right)$, and the function $\eta$ that controls the quasisymmetry of $g$.

The point here is that $F^{*}$ is serious as a set unto itself, not as a subset of something else.

Before stating the next thickening result we need another definition. 
Definition 1.11. A closed set $E$ of $\mathbb{R}^{n}$ is said to be a strong set if there is a constant $C>0$ so that for each $x \in \mathbb{R}^{n} \backslash E$ there is a $y \in E$ such that

$$
|x-y| \leq C \operatorname{dist}(x, E)
$$

and

$$
\operatorname{dist}\left(y, \mathbb{R}^{n} \backslash E\right) \geq C^{-1} \operatorname{dist}(x, E) .
$$

In other words, a strong set is always approximately at least as big as its complement.

Proposition 1.14. Suppose that $F$ is a serious closed subset of $\mathbb{R}^{n}$ and that $g: F \rightarrow \mathbb{R}^{n}$ is quasisymmetric. Then there is a strong set $S \subseteq \mathbb{R}^{n}$ such that $S \supseteq F$ and $g$ admits an extension to a quasisymmetric mapping $G: S \rightarrow \mathbb{R}^{n}$. The strongness constant for $S$ and the function which governs the quasisymmetry of $G$ can be chosen to depend only on the function that governs the quasisymmetry of $g$, the seriousness constant for $F$, and the dimension $n$.

We shall need to know that the image is a strong set, and there is a general result to this effect.

Proposition 1.15. If $G: S \rightarrow \mathbb{R}^{n}$ is quasisymmetric and $S$ is a strong subset of $\mathbb{R}^{n}$, then so is $G(S)$, with a constant that depends only on the dimension, the strongness constant for $S$, and the function which governs the quasisymmetry of $G$.

It may not be clear that these statements reflect progress, but the they do, and this is manifested in part by the following fact, which says that strong sets are large measure-theoretically.

Proposition 1.16. If $S$ is a strong subset of $\mathbb{R}^{n}$, then there is a constant $C>0$ so that

$$
|S \cap B(x, r)| \geq C^{-1} r^{n},
$$

for all $x \in S$ and $r>0$. $C$ depends only on the dimension and the strongness constant of $S$. 
Here $|A|$ denotes the Lebesgue measure of a set $A$.

The next point is to convert from mappings to measures. We begin with some definitions.

Definition 1.18. Let $E$ be a closed subset of $\mathbb{R}^{n}$, and let $\mu$ be a Borel measure with support equal to $E$. that

a) We say that $\mu$ is doubling on $E$ if there is a constant $C>0$ so

$$
\mu(B(x, 2 r)) \leq C \mu(B(x, r))
$$

for all $x \in E$ and $0<r<\operatorname{diam} E$.

b) Define $\delta(x, y)=\delta_{\mu}(x, y)$ for $x, y \in E$ by

$$
\delta(x, y)=(\mu(B(x,|x-y|) \cup B(y,|x-y|)))^{1 / n} .
$$

We say that $\mu$ is a metric doubling measure on $E$ if $\mu$ is doubling on $E$ and if there is a true metric $d(x, y)$ on $E$-i.e., a symmetric nonnegative function which vanishes exactly on the diagonal and which satisfies the triangle inequality - and a constant $C>0$ such that

$$
C^{-1} d(x, y) \leq \delta(x, y) \leq C d(x, y),
$$

for all $x, y \in E$.

These are good classes of measures for studying quasisymmetric mappings. The notion of metric doubling measures comes from [DS], in a slightly different form, see also [S1].

Proposition 1.22. If $G: S \rightarrow \mathbb{R}^{n}$ is quasisymmetric and $S$ is a strong subset of $\mathbb{R}^{n}$, then the measure $\mu$ on $\mathbb{R}^{n}$ defined by $\mu(A)=|G(A \cap S)|$ is a metric doubling measure on $S$, with constants that depend only on $n$, the strongness constant for $S$, and the function which governs the quasisymmetry of $G$.

This is exactly the measure that we are interested in for Theorem 1.3. The question now is what more we can say about it.

Proposition 1.23. If $S$ is a strong subset of $\mathbb{R}^{n}$ and $\mu$ is a metric doubling measure on $S$, then there is a metric doubling measure $\nu$ on $\mathbb{R}^{n}$ which agrees with $\mu$ on subsets of $S$. The metric doubling constants 
for $\nu$ are controlled in terms of the corresponding constants for $\mu$, the strongness constant for $S$, and the dimension $n$.

This is what we want because of the following absolute continuity result.

Theorem 1.24. If $\mu$ is a metric doubling measure on $\mathbb{R}^{n}$ and $n>1$, then $\mu$ and Lebesgue measure are absolutely continuous with respect to each other.

This result was basically proved by Gehring [G]. He did not state it this way, but his argument gives this result with little extra effort. This extension of Gehring's result was observed in [DS]. See Proposition 3.4 of [S1] for a detailed argument for this form of the result.

If $\mu$ is a metric doubling measure on $\mathbb{R}^{n}, n>1$, then the density of $\mu$ is an " $A_{\infty}$ weight", which gives a uniform and scale-invariant version of absolute continuity. In other words Theorem 1.24 comes with quantitative estimates.

The original point of Gehring's argument was to get information about the jacobian of a global quasisymmetric mapping on $\mathbb{R}^{n}$. We are doing roughly the same thing here, except that we are exploiting some flexibility in metric doubling measures that quasisymmetric mappings do not enjoy. Specifically, in Proposition 1.23 we have an extension result which does not have a counterpart for quasisymmetric mappings. There are no topological obstructions to building extensions of metric doubling measures.

Not all metric doubling measures on $\mathbb{R}^{n}$ arise from global quasisymmetric mappings in the manner described above. See [S2] for counterexamples.

Let us now summarize some of the main conclusions of these propositions.

Theorem 1.25. Suppose that $F$ is a closed subset of $\mathbb{R}^{n}$, that $g: F \rightarrow$ $\mathbb{R}^{n}$ is quasisymmetric, and that $F_{0}$ is a closed serious subset of $F$. Then there is a metric doubling measure $\nu$ on $\mathbb{R}^{n}$ such that $\nu(A)=|g(A)|$ for all Borel subsets of $F_{0}$. In particular $|g(A)|=0$ if and only if $|A|=0$ when $A \subset F_{0}$, by Theorem 1.24. The metric doubling constants for $\nu$ depend only on $n$, the seriousness constant for $\left(F_{0}, F\right)$, and the function which governs the quasisymmetry of $g$. 
Indeed, under these conditions we can use Proposition 1.10 to extend the restriction of $g$ to $F_{0}$ to a quasisymmetric mapping on a serious set, and then we can use Proposition 1.14 to extend to a strong set. This permits us to reduce to the case where $F_{0}$ is a strong set. We then use Propositions 1.22 and 1.23 to get a metric doubling measure, first on the strong set, and then on all of $\mathbb{R}^{n}$. This proves Theorem 1.25, modulo the previous propositions.

Theorem 1.3 is an immediate consequence of Lemma 1.8 and Theorem 1.25. Thus we need only prove the various propositions. They are slightly messy, but all pretty straightforward, and largely implicit in the literature, if not explicitly stated in the form that we need. For the sake of readability we shall often provide more detail than needed for experts in the area, and we shall sometimes treat issues with bare hands instead of sending the reader to the literature for lemmata.

Related papers concerning quasisymmetric mappings include [TV], [V2], and [V3].

Although Propositions 1.10 and 1.14 look very similar, they really aren't, in the sense that Proposition 1.10 is much closer to the definitions, whereas the proof of Proposition 1.14 relies on the structure of Euclidean space.

\section{The proof of Proposition 1.10.}

This is quite straightforward. We are going to take $F_{0}$, take a reasonably dense but scattered subset of $F \backslash F_{0}$, replace $g$ by something simple on little disks centered at points in this scattered subset, and that will do the job. Our first task is to find this reasonably dense but scattered subset. We shall employ this well-known construction again in the next section.

Lemma 2.1. Let $E$ be a closed subset of $\mathbb{R}^{n}$, and let $H$ be a subset of $\mathbb{R}^{n} \backslash E$. Then we can find a subset $I$ of $H$ such that

for every $x \in H$ there is a point $u \in I$ such that

$$
|x-u| \leq \frac{1}{2} \operatorname{dist}(x, E),
$$

and

(2.3) for every $y, z \in I$ we have that $|y-z| \geq \frac{1}{3} \operatorname{dist}(y, E)$. 
Thus $I$ is reasonably dense in $H$ and also reasonably scattered.

Let $E$ and $H$ be given, and let $I$ be a maximal subset of $H$ which satisfies (2.3). It is not hard to find such a maximal subset. For instance, one can write $\mathbb{R}^{n} \backslash E$ as the increasing union of compact sets $K_{j}$, one can build sets $I_{j}$ recursively by taking $I_{j+1}$ to be the maximal subset of $H \cap K_{j+1}$ which satisfies (2.3) and contains $I_{j}$, and then take $I$ to be the union of the $I_{j}$ 's. In each compact part the maximal subset has to be finite, which makes it easier to verify its existence, and then the pieces nest together properly to give maximality for the union.

Thus we can take $I$ to be a maximal subset of $H$ which satisfies (2.3). Let $x \in H$ be given. Either $x \in I$ already, or it is not, in which case $I \cup\{x\}$ will not satisfy (2.3). This means that there is a point $u \in I$ such that

(2.4) either $\quad|x-u|<\frac{1}{3} \operatorname{dist}(x, E) \quad$ or $\quad|x-u|<\frac{1}{3} \operatorname{dist}(u, E)$.

In the first case we get (2.2) directly. In the second case we compute that

$$
\operatorname{dist}(u, E) \leq|x-u|+\operatorname{dist}(x, E) \leq \frac{1}{3} \operatorname{dist}(u, E)+\operatorname{dist}(x, E)
$$

to conclude that dist $(u, E) \leq 3 \operatorname{dist}(x, E) / 2$, and hence that $(2.2)$ holds. This proves Lemma 2.1.

Lemma 2.6. Let $E, H$, and $I$ be as in Lemma 2.1, and set $B(x)=$ $\bar{B}\left(x, 20^{-1}\right.$ dist $\left.(x, E)\right)$ when $x \in I$. If $x \in I$ and $y \in 2 B(x)$, then

$$
\frac{9}{10} \operatorname{dist}(x, E) \leq \operatorname{dist}(y, E) \leq \frac{11}{10} \operatorname{dist}(x, E) .
$$

If $x, z \in I$ and $x \neq z$ then

$$
2 B(x) \cap 2 B(z)=\varnothing .
$$

Indeed, if $x \in I$ and $y \in 2 B(x)$, then

$$
|\operatorname{dist}(y, E)-\operatorname{dist}(x, E)| \leq 10^{-1} \operatorname{dist}(x, E) \text {. }
$$


This implies (2.7).

Now suppose that $x, z \in I$ and $x \neq z$, but that (2.8) fails to hold, so that there is a point $y$ in the intersection. Then

$$
|x-z| \leq|x-y|+|y-z| \leq \frac{1}{10} \operatorname{dist}(x, E)+\frac{1}{10} \operatorname{dist}(z, E)
$$

and

$$
\operatorname{dist}(z, E) \leq \frac{10}{9} \operatorname{dist}(y, E) \leq \frac{11}{9} \operatorname{dist}(x, E),
$$

because of (2.7) (applied to both $x$ and $z$ ). Combining these we get that

$$
|x-z|<\frac{1}{3} \operatorname{dist}(x, E)
$$

in contradiction to (2.3). This proves (2.8), and the lemma follows.

Let us now prove Proposition 1.10. Let $g, F, F_{0}$ be as given there, and apply Lemma 2.1 with $E=F_{0}$ and $H=F \backslash F_{0}$. We get a subset $I$ of $F$.

Define $F^{*}$ by

$$
F^{*}=F_{0} \cup\left(\bigcup_{x \in I} B(x)\right)
$$

where $B(x)$ is as in Lemma 2.6, with $E=F_{0}$. We shall define $g^{*}$ a little later. Let us first verify some simple properties of $F^{*}$.

Lemma 2.14. $F^{*}$ is closed.

Let $\left\{z_{j}\right\}$ be a sequence of points in $F^{*}$ which converges to some point $z \in \mathbb{R}^{n}$. We have to show that $z \in F^{*}$. If there is a subsequence of $\left\{z_{j}\right\}$ which is contained in $F_{0}$, then $z \in F_{0}$, and $z \in F^{*}$. If $\left\{z_{j}\right\}$ has a subsequence which is contained in any one of the $B(x)$ 's, then $z$ lies in the same $B(x)$, and hence in $F^{*}$. The remaining possibility is that there is a subsequence of $\left\{z_{j}\right\}$ such that each term lies in a different $B(x)$. Since $\left\{z_{j}\right\}$ converges and hence is bounded, we must have that the elements of this subsequence accumulate on $F_{0}$, because of the way that we defined the $B(x)^{\prime}$ 's. In this case we conclude that $z \in F_{0}$ and hence $z \in F^{*}$. This proves the lemma. 
Lemma 2.15. $\operatorname{diam} F^{*} \leq 2 \operatorname{diam} F$.

If $p \in F^{*}$, then either $p \in F_{0} \subseteq F$, or $p \in B(x)$ for some $x \in I$. In the latter case we have that

$$
\operatorname{dist}(p, F) \leq|p-x| \leq 10^{-1} \operatorname{dist}\left(x, F_{0}\right) \leq 10^{-1} \operatorname{diam} F,
$$

since $x \in F$. This implies the desired bound for $\operatorname{diam} F^{*}$.

Lemma 2.17. For each point $x \in F$ there is a point $u \in F^{*}$ such that $|x-u| \leq \operatorname{dist}\left(x, F_{0}\right) / 2$.

This is trivial. Either $x \in F_{0}$, in which case we take $u=x$, or not, in which case we take $u \in I$ as in (2.2) (with $E=F_{0}$ ). This gives the lemma.

Lemma 2.18. $F^{*}$ is serious.

Let $p \in F^{*}$ and $0<t<\operatorname{diam} F^{*}$ be given, and let us try to find a point $q \in F^{*}$ with

$$
C^{-1} t \leq|p-q| \leq C t
$$

for a suitable constant $C$. We may as well assume that $t \leq \operatorname{diam} F$, since otherwise we can use Lemma 2.15 to reduce the problem to the definition of $\operatorname{diam} F^{*}$.

Suppose first that $p \in F_{0}$. The we can use the assumption that $F_{0}$ is a serious subset of $F$ to find a point $x \in F$ such that $C^{-1} t \leq|p-x| \leq t$. Lemma 2.17 provides a point $u \in F^{*}$ such that $|x-u| \leq \operatorname{dist}\left(x, F_{0}\right) / 2 \leq$ $|p-x| / 2$. Thus $|p-u| \leq|p-x|+|x-u| \leq 2 t$, which gives the upper bound in (2.19) (with $q=u$ ). For the lower bound we have that

$$
|p-x| \leq|p-u|+|u-x| \leq|p-u|+\frac{1}{2}|x-p|,
$$

and hence $|p-x| / 2 \leq|p-u|$. This gives the lower bound in (2.19).

Now suppose that $p \in B(z)$ for some $z \in I$. If $t \leq \operatorname{dist}\left(z, F_{0}\right)$, then we can find the required $q$ inside $B(z)$. If $t>\operatorname{dist}\left(z, F_{0}\right)$, then let $y$ be a point in $F_{0}$ such that $|y-z|=\operatorname{dist}\left(z, F_{0}\right)$. Choose $x \in F$ so that $C^{-1} t \leq|y-x| \leq t$, as we can do because of the seriousness of $F_{0}$ inside of $F$. Let $u \in F^{*}$ be associated to $x$ as in Lemma 2.17. Then

$$
\begin{aligned}
|p-u| & \leq|p-z|+|z-y|+|y-x|+|x-u| \\
& \leq t+t+t+\operatorname{dist}\left(x, F_{0}\right) \\
& \leq 3 t+|x-y| \leq 4 t .
\end{aligned}
$$


This gives the upper bound that we want for (2.19) (with $q=u$ ). For the lower bound we observe that

$$
\begin{aligned}
|y-x| & \leq|p-u|+|p-y|+|x-u| \\
& \leq|p-u|+(|p-z|+|z-y|)+\frac{1}{2} \operatorname{dist}\left(x, F_{0}\right) \\
& \leq|p-u|+\operatorname{dist}\left(z, F_{0}\right)+\operatorname{dist}\left(z, F_{0}\right)+\frac{1}{2}|x-y| \\
& \leq|p-u|+2 \operatorname{dist}\left(z, F_{0}\right)+\frac{1}{2}|x-y| .
\end{aligned}
$$

Thus $C^{-1} t \leq|y-x| \leq 2|p-u|+4 \operatorname{dist}\left(z, F_{0}\right)$. If $t$ is much larger that $\operatorname{dist}\left(z, F_{0}\right)$ then this implies the lower bound in (2.19). If not, then again we simply take a suitable $q$ in $B(z)$. This proves Lemma 2.18.

Let us now define $g^{*}: F^{*} \rightarrow \mathbb{R}^{n}$. Of course we set $g^{*}=g$ on $F_{0}$, and we define $g^{*}$ on each $B(x)$ as follows. Given $x \in I$ choose a point $\pi(x) \in F_{0}$ so that

$$
|x-\pi(x)|=\operatorname{dist}\left(x, F_{0}\right)
$$

Define $g^{*}$ on $B(x)$ by

$$
g^{*}(w)=g(x)+a \frac{|g(x)-g(\pi(x))|}{|x-\pi(x)|}(w-x),
$$

for all $w \in B(x)$. Here $a$ is a small positive number to be chosen in the next lemma. Thus on the ball $B(x)$ we have taken $g^{*}$ to be a similarity with the same value as $g$ at the center and whose distortion ratio is approximately the same as that of $g$ at that location and scale.

Let $\beta(x), x \in I$, denote the ball which is the image of $B(x)$ under $g^{*}$. Thus

$$
\beta(x)=B\left(g(x), 20^{-1} a|g(x)-g(\pi(x))|\right),
$$

by the definition of $g^{*}$ and $B(x)$ (in Lemma 2.6). 
Lemma 2.26. If $a$ is small enough, depending only on the function which governs the quasisymmetry of $g$, then the balls $2 \beta(x), x \in I$, are pairwise disjoint and each is disjoint from $g^{*}\left(F_{0}\right)=g\left(F_{0}\right)$.

This is just a question of the quasisymmetry condition. Suppose that $y, z \in I, y \neq z$. Using (2.3) we get that $|y-\pi(y)| \leq 3|y-z|$, and similarly we have that $|z-\pi(z)| \leq 3|y-z|$. Quasisymmetry then implies that

$$
|g(y)-g(\pi(y))|+|g(z)-g(\pi(z))| \leq C|g(y)-g(z)| .
$$

This implies that $2 \beta(y)$ and $2 \beta(z)$ are disjoint if $a$ is small enough.

Now suppose that $x \in I$ and $w \in F_{0}$. We want to show that $g(w) \notin 2 \beta(x)$. We have that $|x-\pi(x)|=\operatorname{dist}\left(x, F_{0}\right) \leq|x-w|$, by definition of $\pi(x)$, and so

$$
|g(x)-g(\pi(x))| \leq C|g(x)-g(w)|,
$$

by quasisymmetry. This implies that $g(w) \notin 2 \beta(x)$ if $a$ is small enough.

This proves Lemma 2.26. Fix now a choice of $a$ as above, depending only on the function that governs the quasisymmetry of $g$.

It remains to prove that $g^{*}$ is quasisymmetric. The argument for this has some generality, and we shall need it again later, and so we formulate it in more general terms than required for the present circumstances.

Lemma 2.29. Let $A$ be a closed subset of $\mathbb{R}^{n}$, and let $\left\{B_{i}\right\}_{i \in I}$ and $\left\{\beta_{i}\right\}_{i \in I}$ be collections of closed balls in $\mathbb{R}^{n}$. Set $A^{*}=A \cup \bigcup_{i \in I} B_{i}$ and let $A^{\prime}$ denote the union of $A$ and the set of centers of the balls $B_{i}, i \in I$.

Suppose that $H: A^{*} \rightarrow \mathbb{R}^{n}$ has the property that the restriction of $H$ to $A^{\prime}$ is quasisymmetric, that $H\left(B_{i}\right)=\beta_{i}$ for each $i \in I$, and that the restriction of $H$ to each $B_{i}$ is a quasisymmetric mapping with a function governing the quasisymmetry that can be taken to be independent of $i$. Suppose also that the balls $2 B_{i}, i \in I$, are pairwise disjoint and are disjoint from $A$, that the balls $2 \beta_{i}, i \in I$, are pairwise disjoint and disjoint from $H(A)$, and that there is a constant $C>0$ so that

$$
C^{-1} \operatorname{dist}\left(B_{i}, A\right) \leq \operatorname{radius} B_{i} \leq \operatorname{dist}\left(B_{i}, A\right),
$$

and

$$
C^{-1} \operatorname{dist}\left(\beta_{i}, H(A)\right) \leq \operatorname{radius} \beta_{i} \leq \operatorname{dist}\left(\beta_{i}, H(A)\right)
$$


for all $i \in I$. (Note that the upper bounds follow from the disjointness of the $2 B_{i}$ 's from $A$, the $2 \beta_{i}$ 's from $H(A)$.)

Then $H: A^{*} \rightarrow \mathbb{R}^{n}$ is quasisymmetric, with bounds which depend only on a uniform choice of a function which governs the quasisymmetry of the various restrictions of $H$ mentioned above, and on the constants in $(2.30)$.

If we can prove this lemma then we get that $g^{*}$ is quasisymmetric, because our balls have the correct disjointness properties and satisfy the analogue of (2.30) (by their definitions), because the restrictions of $g^{*}$ to the various $B(x)$ 's are trivially quasisymmetric, with uniform bounds, and because the restriction of $g^{*}$ to $A^{\prime}=F_{0} \cup I$ agrees with $g$ and hence is quasisymmetric.

Thus Proposition 1.10 will follow once we prove Lemma 2.29.

Beware of the small changes in notation from the previous situation to the lemma, $B(x)$ to $B_{i}$, etc.

The lemma is a straightforward but unpleasant exercise, a matter of checking cases. Let $A, H$, etc. be as above.

Let us first record a small observation.

Sublemma 2.31. Suppose that $p, q \in B_{i}$ and $w \in A^{*} \backslash B_{i}$. Then

$$
\begin{gathered}
C^{-1}|q-w| \leq|p-w| \leq C|q-w| \\
C^{-1}|H(q)-H(w)| \leq|H(p)-H(w)| \leq C|H(q)-H(w)|, \\
|p-w| \geq C^{-1} \operatorname{diam} B_{i}
\end{gathered}
$$

and

$$
|H(p)-H(w)| \geq C^{-1} \operatorname{diam} \beta_{i},
$$

for a suitable constant $C$.

This follows from the our assumptions, which ensure that $2 B_{i}$ is disjoint from $A^{*} \backslash B_{i}$, and that $2 \beta_{i}$ is disjoint from $H\left(A^{*} \backslash B_{i}\right)$.

Suppose that we are given $x, y, z \in A^{*}$ and $t>0$ which satisfy $|x-y| \leq t|x-z|$. We want to show that $|H(x)-H(y)| \leq \theta(t) \mid H(x)-$ $H(z) \mid$ for some $\theta(t)$ which tends to 0 when $t \rightarrow 0$ and which is bounded 
on finite intervals. (See Lemma 2.42 below for a small technical point here.)

If $x, y, z$ all lie in $A^{\prime}$, or all lie in some $B_{i}$, then we get the desired bound from our hypotheses.

If no two of $x, y, z$ lie in the same $B_{i}$, then we can reduce to the previous case where $x, y, z$ all lie in $A^{\prime}$, by using Sublemma 2.31 to switch from a point in some $B_{i}$ to the center of $B_{i}$. That is, such a change will not affect any of the distances involved by more than a bounded factor. (Remember that $A^{\prime}$ consists exactly of $A$ and the various centers of the $B_{i}$ 's.)

Thus we may assume that exactly two of $x, y, z$ lie in some $B_{i}$, and that the remaining point lies in $A^{*} \backslash B_{i}$.

We may as well assume that $x$ is one of the two points that lies in $B_{i}$. For if it is not, then we can use Sublemma 2.31 to reduce to the case where $y$ and $z$ are both equal to the center of $B_{i}$, and where $x$ either lies in $A$ or is the center of some other $B_{j}$. Again these changes will not affect the relevant distances by more than a bounded factor. After these changes all three points would lie in $A^{\prime}$, which is already covered by our assumptions.

Thus we may assume that $x$ lies in $B_{i}$, and that exactly one of $y$ and $z$ do too. We may also assume that the remaining point lies in $A^{\prime}$, because Sublemma 2.31 again permits us to make the substitution without affecting the quantities involved by more than a bounded factor.

In order to deal with this remaining situation we make another small observation.

Sublemma 2.36. For each $i \in I$ let $c_{i}$ denote the center of $B_{i}$, and choose $\xi_{i} \in A$ such that $\left|c_{i}-\xi_{i}\right|=\operatorname{dist}\left(c_{i}, A\right)$. Then

$$
C^{-1} \operatorname{diam} B_{i} \leq\left|c_{i}-\xi_{i}\right| \leq C \operatorname{diam} B_{i}
$$

and

$$
C^{-1} \operatorname{diam} \beta_{i} \leq\left|H\left(c_{i}\right)-H\left(\xi_{i}\right)\right| \leq C \operatorname{diam} \beta_{i}
$$

for each $i$ and a suitable constant $C$.

The bounds (2.37) follow from (2.30) and the definitions of $c_{i}$ and $\xi_{i}$. (See also Sublemma 2.31.) 
As for (2.38), notice that $\left|H\left(c_{i}\right)-H\left(\xi_{i}\right)\right|$ is comparable in size to $\operatorname{dist}\left(H\left(c_{i}\right), H(A)\right)$, because of quasisymmetry and the fact that $\mid c_{i}-$ $\xi_{i} \mid=\operatorname{dist}\left(c_{i}, A\right)$. This implies (2.38), because of (2.30) again.

This proves Sublemma 2.36.

Let us come back to our original problem of the quasisymmetry of $H$. We have our three points $x, y, z$ with $|x-y| \leq t|x-z|$, and we want to prove something like $|H(x)-H(y)| \leq \theta(t)|H(x)-H(z)|$. We have already reduced to the case where $x$ and exactly one of $y$ and $z$ lies in some $B_{i}$, and where the remaining point lies in $A^{\prime} \backslash B_{i}$.

This last situation is slightly obnoxious because it is really a combination of two cases. For the sake of explanation suppose that it is $y$ which lies in $B_{i}$. Then we could have that $|x-y|$ is very small compared to the radius of $B_{i}$, and that dist $\left(z, B_{i}\right)$ is large compared to the radius of $B_{i}$. In order to establish quasisymmetry we should show that such a circumstance leads to something similar in the image. It is more convenient however to do this in two steps, first to compare $|x-y|$ with the radius of $B_{i}$ and make a similar comparison in the image, and then to compare dist $\left(z, B_{i}\right)$ with the radius of $B_{i}$ and to make a similar comparison in the image. Our final estimate will be obtained as a product of estimates from these two parts.

Assume first that $y \in B_{i}$, so that $z \in A^{\prime} \backslash B_{i}$. Set

$$
\begin{aligned}
r & =\frac{|x-y|}{\left|c_{i}-\xi_{i}\right|}, & s & =\frac{\left|c_{i}-\xi_{i}\right|}{|x-z|}, \\
R & =\frac{|H(x)-H(y)|}{\left|H\left(c_{i}\right)-H\left(\xi_{i}\right)\right|}, & S & =\frac{\left|H\left(c_{i}\right)-H\left(\xi_{i}\right)\right|}{|H(x)-H(z)|} .
\end{aligned}
$$

By assumption we have that $r s \leq t$, and we want to bound $R S$ by a function of $t$ which tends to 0 as $t \rightarrow 0$.

Sublemma 2.40. $r, s, R, S \leq C$ for some constant $C$.

For $r$ and $R$ this follows from Sublemma 2.36 and the fact that $x, y \in B_{i}, H(x), H(y) \in \beta_{i}$. For $s$ and $S$ we observe that $z \notin B_{i}$, $H(z) \notin \beta_{i}$, so that Sublemma 2.31 can be applied. With this observation the bounds for $s$ and $S$ follow from Sublemma 2.36 also. This proves Sublemma 2.40.

Since $r s \leq t$ we get that one of $r$ and $s$ is $\leq \sqrt{t}$. Our quasisymmetry hypotheses imply that the corresponding $R$ or $S$ is bounded by 
a good function of $\sqrt{t}$. (For $r$ we have to use (2.37) to get to the quasisymmetry of $H$ on $B_{i}$.) We conclude that $R S$ is bounded by a good function of $\sqrt{t}$, since they are each bounded separately. This is the bound that we need.

Assume now that $z \in B_{i}$, so that $y \in A^{\prime} \backslash B_{i}$. Define $r, s, R, S$ as above. Again we have $r s \leq t$ by assumption, and we want to control $R S$.

Sublemma 2.41. $r, s, R, S \geq C^{-1}$ for some constant $C$.

This is practically the same as Sublemma 2.40, but with the roles of $y$ and $z$ reversed.

In this case we can conclude that each of $r$ and $s$ is bounded by a constant multiple of $t$. Our quasisymmetry hypotheses then imply that each of $R$ and $S$ is bounded by a function of $t$, and so the product is too.

This completes the proof of Lemma 2.29. Note that we have not given the most efficient estimates in the argument.

For the record, let us mention a small lemma which we have used implicitly.

Lemma 2.42. Suppose that $\theta:[0, \infty) \rightarrow[0, \infty)$ satisfies $\theta(0)=0, \theta(t)$ is continuous at 0 , and $\theta$ is bounded on bounded sets. Then there is a homeomorphism $\Theta:[0, \infty) \rightarrow[0, \infty)$ such that $\theta(t) \leq \Theta(t)$ for all $t$.

Indeed, following Väisälä we set $\Theta(t)=t+\sup _{0 \leq s \leq 2 t} \theta(t)$ when $t=2^{n}, n \in \mathbb{Z}$, and use affine interpolation to define $\Theta$ on the rest. (Thanks to Alestalo for pointing out the author's stupidity for the first version.)

\section{The proof of Proposition 1.14.}

The argument will parallel the proof of Proposition 1.10 in the previous section, except for one piece of information that we shall have to obtain for ourselves.

Let $g$ and $F$ be given, as in Proposition 1.14. Let $I$ be as in Lemma 2.1, applied with $E=F$ and $H=\mathbb{R}^{n} \backslash F$. Let $B(x), x \in I$, be defined 
as in Lemma 2.6 (with $E=F$ ). Define the set $S$ by

$$
S=F \cup\left(\bigcup_{x \in I} B(x)\right)
$$

as in (2.13).

In the next two lemmas we give basic properties of $S$. At this stage we do not use the assumption that $F$ is serious, only that it is closed. The seriousness will not be used until we start to work with our quasisymmetric mapping.

Lemma 3.2. $S$ is closed.

This is the same as Lemma 2.14, with only cosmetic changes.

Lemma 3.3. $S$ is a strong set.

Let $x \in \mathbb{R}^{n} \backslash S$ be given, as in Definition 1.11. Thus $x \in \mathbb{R}^{n} \backslash F$. The point is that $x$ must be reasonably close to $B(u)$ for some $u \in I$, but it is helpful to distinguish between the cases where $x$ is very close to some $B(u)$ or never too close. Actually our threshold will be sufficiently generous that the latter never happens.

Suppose first that

$$
\operatorname{dist}(x, S) \leq \frac{1}{2} \operatorname{dist}(x, F)
$$

Choose $z \in S$ so that $|x-z|=\operatorname{dist}(x, S)$. Then $z \notin F$, and so $z \in B(u)$ for some $u \in I$. Because $|x-z| \leq \operatorname{dist}(x, F) / 2$ we get that

$$
\frac{1}{2} \operatorname{dist}(x, F) \leq \operatorname{dist}(z, F) \leq \frac{3}{2} \operatorname{dist}(x, F) \text {. }
$$

This means that dist $(z, F)$ is comparable in size to the radius of $B(u)$, because of (2.7) in Lemma 2.6 and the definition of $B(u)$. Since $|x-z|=$ dist $(x, S)$ and $z \in B(u)$ it is easy to see that we can find a point $y$ of the type required in Definition 1.11, inside $B(u)$ (and not just in $S$ ).

Now suppose that

$$
\operatorname{dist}(x, S)>\frac{1}{2} \operatorname{dist}(x, F) \text {. }
$$


In fact this cannot happen. Indeed, apply Lemma 2.1 to get a point $u \in I$ such that $|x-u| \leq \operatorname{dist}(x, F) / 2$, as in (2.2). Then

$$
\operatorname{dist}(x, S)<|x-u| \leq \frac{1}{2} \operatorname{dist}(x, F)<\operatorname{dist}(x, S),
$$

a contradiction.

This completes the proof of Lemma 3.3.

To prove Proposition 1.14 we need to build a quasisymmetric extension $G$ of $g$. We would like to do this in the same way as in Section 2 (around (2.24)), but in the present situation we have the problem that $g$ is not yet defined at the elements of $I$. The main point of the argument that follows will be to extend $g$ quasisymmetrically to $I$. Once we do that we can proceed as in Section 2 (using Lemma 2.29).

The elements of $I$ basically represent holes in $F$, large puddles of its complement. We need to show that these holes correspond to holes in the complement of $g(F)$ in a reasonable manner. The next couple of lemmas will enable us to do that.

Lemma 3.8. Let a homeomorphism $\eta:[0, \infty) \rightarrow[0, \infty)$ and a dimension $n$ be given. For each $\varepsilon>0$ there exist $\delta>0$ and $R>1$, depending on $\varepsilon, \eta$, and $n$, with the following properties. Let $E$ be a subset of $\mathbb{R}^{n}$ and $h: E \rightarrow \mathbb{R}^{n}$ be an $\eta$-quasisymmetric mapping which satisfy the normalizations

$$
0, u \in E \quad \text { and } \quad h(0)=0, h(u)=u,
$$

where $u=(1,0, \ldots, 0)$. Suppose that $E$ is $\delta$-thick in $B(0, R)$, in the sense that

$$
\operatorname{dist}(x, E) \leq \delta \quad \text { whenever } x \in B(0, R) .
$$

Then $h(E \cap B(0, R))$ is $\varepsilon$-thick in $B(0,1)$, so that

$$
\operatorname{dist}(z, h(E)) \leq \varepsilon \quad \text { whenever } z \in B(0,1) \text {. }
$$

This is a weaker version of [V3, Theorem 3.1], weaker by dint of having estimates which depend on the dimension and which are obtained through very nonconstructive means. For the reader's convenience we include a proof by compactness which is mentioned in the introduction of [V3] (and attributed to Tukia). 
Suppose that Lemma 3.8 is not true. Then there exist $\eta, n$, and $\varepsilon$ as above, a sequence $\left\{E_{k}\right\}$ of subsets of $\mathbb{R}^{n}$, and a sequence $\left\{h_{k}\right\}$ of $\eta$-quasisymmetric mappings from $E_{k}$ into $\mathbb{R}^{n}$, such that $\left\{E_{k}\right\}$ and $\left\{h_{k}\right\}$ satisfy the analogues of the normalizations (3.9), each $E_{k}$ is $1 / k$ thick inside $B(0, k)$, but each $h_{k}\left(E_{k} \cap B(0, k)\right)$ fails to be $\varepsilon$-thick inside $B(0,1)$.

Claim 3.12. There is a sequence of integers $k_{j}$ and an $\eta$-quasisymmetric mapping $H: \mathbb{R}^{n} \rightarrow \mathbb{R}^{n}$ such that the $h_{k_{j}}$ 's converge to $H$ "uniformly on compact sets" in the sense that

$$
\lim _{j \rightarrow \infty} \sup _{x \in B \cap E_{k_{j}}}\left|h_{k_{j}}(x)-H(x)\right|=0,
$$

for every ball $B$ in $\mathbb{R}^{n}$.

This is pretty standard, but let us be careful.

The first step is to show that we have equicontinuity of the $h_{k}$ 's on compact sets. That is, for each ball $B$ there exists a function $\omega_{B}$ : $[0, \infty) \rightarrow[0, \infty)$ such that $\omega_{B}(0)=0, \omega_{B}$ is continuous at $0, \omega_{B}$ is bounded on finite intervals, and

$$
\left|h_{k}(x)-h_{k}(y)\right| \leq \omega_{B}(|x-y|),
$$

for all $x, y \in B \cap E_{k}$ and all $k$. This follows from the uniform quasisymmetry hypotheses and the normalizations.

Once we have this equicontinuity condition we can conclude that there is continuous mapping $H: \mathbb{R}^{n} \rightarrow \mathbb{R}^{n}$ and a subsequence $\left\{h_{k_{j}}\right\}$ of $\left\{h_{k}\right\}$ which converges to $h$ in the sense of (3.13). This is not hard to prove, using an Arzela-Ascoli argument. Here is one way to do it from scratch. Let $\left\{p_{m}\right\}$ be a countable dense subset of $\mathbb{R}^{n}$. For each $p_{m}$ choose a sequence of points $\left\{p_{m, k}\right\}_{k=1}^{\infty}$ such that $p_{m, k} \in E_{k}$ for each $k$ and $\left|p_{m, k}-p_{m}\right| \leq 1 / k$ when $p_{m} \in B(0, k)$. We can do this because of our thickness hypotheses. Next choose the subsequence $\left\{h_{k_{j}}\right\}$ of $\left\{h_{k}\right\}$ in such a way that $\lim _{j \rightarrow \infty} h_{k_{j}}\left(p_{m, k_{j}}\right)$ exists for each $m$, and call the result $H\left(p_{m}\right)$. We can find such a subsequence because of the usual Cantor diagonalization argument. We are also using our normalizations and the equicontinuity property (3.14) to know that $\left\{h_{k}\left(p_{m, k}\right)\right\}_{k}$ is a bounded sequence for each $m$. Once one has $\left\{h_{k_{j}}\right\}$ with this property it is not hard to show that $H$ must have a continuous extension to all of $\mathbb{R}^{n}$, and that we have convergence in the sense of (3.13), using the equicontinuity property (3.14). 
It is easy to derive the $\eta$-quasisymmetry of $H$ from the corresponding property of the $h_{k}$ 's.

This completes the proof of Claim 3.12.

Let us now finish the proof of Lemma 3.8. Let $H$ be as in the claim. The main point now is that $H$ must be surjective, $H\left(\mathbb{R}^{n}\right)=\mathbb{R}^{n}$. This is well-known (a consequence of invariance of domain and the connectedness of $\mathbb{R}^{n}$. One does not really need $H$ to be quasisymmetric here, it is enough for $H$ to be proper). On the other hand we are assuming that $h_{k}\left(E_{k}\right)$ fails to be $\varepsilon$-thick inside $B(0,1)$ for each $k$. It is not hard to derive a contradiction to this assumption. Indeed, let $k$ be large, to be chosen soon, and suppose that $z_{k} \in B(0,1)$ satisfies

$$
\operatorname{dist}\left(z_{k}, h_{k}\left(E_{k} \cap B(0, k)\right)\right) \geq \varepsilon \text {. }
$$

Because $H$ is a surjection there is a point $x_{k} \in \mathbb{R}^{n}$ such that $H\left(x_{k}\right)=z_{k}$. In fact we have that $x_{k} \in B(0, L)$ for some large $L$ and all $k$, because $H$ is $\eta$-quasisymmetric, and because $z_{k} \in B(0,1)$ for all $k$. In particular we have that $x_{k} \in B(0, k)$ for large enough $k$. For sufficiently large $k$ we can find a point $y_{k} \in E_{k} \cap B(0, L+1)$ such that $\left|x_{k}-y_{k}\right| \leq 1 / k$, because of the thickness property. If $k$ is large and among the $k_{j}$ 's then

$$
\begin{aligned}
\left|z_{k}-h_{k}\left(y_{k}\right)\right| & =\left|H\left(x_{k}\right)-h_{k}\left(y_{k}\right)\right| \\
& \leq\left|H\left(x_{k}\right)-H\left(y_{k}\right)\right|+\left|H\left(y_{k}\right)-h_{k}\left(y_{k}\right)\right|<\varepsilon
\end{aligned}
$$

because of the uniform continuity of $H$ on $B(0, L+1)$ and the uniform convergence (3.13). This contradicts (3.15), and Lemma 3.8 follows.

For our purposes the following reformulation of Lemma 3.8 will be more convenient.

Lemma 3.17. Let a homeomorphism $\eta:[0, \infty) \rightarrow[0, \infty)$, a dimension $n$, and a number $A>1$ be given. Suppose that $X$ is a subset of $\mathbb{R}^{n}$ and that $f: X \rightarrow \mathbb{R}^{n}$ is $\eta$-quasisymmetric. Suppose also that we have $x, y \in X, x \neq y$, and $z \in \mathbb{R}^{n} \backslash X$ such that

$$
|z-x| \leq|x-y| \quad \text { and } \quad \operatorname{dist}(z, X) \geq A^{-1}|x-y| .
$$

Then there is a point $w \in \mathbb{R}^{n} \backslash f(X)$ such that

$$
|w-f(x)| \leq M|f(x)-f(y)|
$$


and

$$
\operatorname{dist}(w, f(X)) \geq M^{-1}|f(x)-f(y)|,
$$

where $M>0$ depends on $\eta, n$, and $A$, but not on anything else.

Roughly speaking, this says that holes in the complement of $X$ correspond under $f$ to holes in the complement of $f(X)$ in a nice way.

This is an easy consequence of Lemma 3.8. We may as well assume that $x=0, y=u=(1,0, \ldots, 0), f(0)=0$, and $f(u)=u$, because we can reduce to that case using affine similarities. We apply Lemma 3.8 with $h=f^{-1}$ and $E=f(X)$. (Lemma 1.4 is relevant here.) More precisely, we argue by contradiction. Suppose that there is no point $w$ as in (3.19), so that $E=f(X)$ is $1 / M$-thick in $B(0, M)$. If $M$ is large enough, then we can apply Lemma 3.8 to conclude that $h(E)=X$ is $\varepsilon$-thick in $B(0,1)$ with $\varepsilon=1 /(2 A)$, for instance. This contradicts our assumption (3.18), and Lemma 3.17 follows.

Let us return now to our earlier story of $F, g$, and $I$. We want to take points in $I$ and associate to them points in the complement of $g(F)$.

Let us decompose $I$ into $I_{0} \cup I_{1}$, where $I_{0}=\{u \in I: \operatorname{dist}(u, F) \leq$ $b \operatorname{diam} F\}, I_{1}=\{u \in I: \operatorname{dist}(u, F)>b \operatorname{diam} F\}$, and $b \in(0,1)$ is a small constant that will be chosen in a moment. $I_{0}$ is the more interesting one, $I_{1}$ can be handled practically without thinking. We shall concern ourselves with only $I_{0}$ for the time being. Note that $I=I_{0}$ and $I_{1}=\varnothing$ when $F$ is unbounded.

Given $u \in I_{0}$, choose points $\pi(u), \rho(u) \in F$ such that

$$
\begin{aligned}
& |u-\pi(u)|=\operatorname{dist}(u, F), \\
& \operatorname{dist}(u, F) \leq|\pi(u)-\rho(u)| \leq C \operatorname{dist}(u, F) .
\end{aligned}
$$

To get $\rho(u)$ we are using our assumption that $F$ is serious. It is here that we choose the constant $b$, once and for all, depending only on the seriousness constant of $F$; we can find such a $\rho(u)$ so long as $\operatorname{dist}(u, F) \leq b \operatorname{diam} F$ and $b$ is small enough. These points $\pi(u), \rho(u)$ are not unique or canonical or anything like that, we simply choose them without worrying about it.

Lemma 3.21. For each $u \in I_{0}$ there is a point $\phi(u) \in \mathbb{R}^{n} \backslash g(F)$ such that

$$
|\phi(u)-g(\pi(u))| \leq C|g(\pi(u))-g(\rho(u))|
$$


and

$$
\operatorname{dist}(\phi(u), g(F)) \geq C^{-1}|g(\pi(u))-g(\rho(u))|
$$

for a suitable constant $C>0$.

This follows from Lemma 3.17, applied with $f=g, X=F, x=$ $\pi(u), y=\rho(u), z=u$, and with $\phi(u)$ taken to be $w$.

For $u \in I_{1}$ we can behave more stupidly.

Lemma 3.24. For each $u \in I_{1}$ we can find a point $\phi(u) \in \mathbb{R}^{n} \backslash g(F)$ such that

$$
\operatorname{dist}(\phi(u), g(F))=\frac{\operatorname{diam} g(F)}{\operatorname{diam} F} \operatorname{dist}(u, F) .
$$

Keep in mind that $\operatorname{dist}(u, F) \geq b \operatorname{diam} F$ this time. This means that there really is no point in choosing something like $\pi(u)$, an element of $F$ closest to $u$, because they are all about the same. It is just a question of the distance to $F$.

The lemma is easy to prove, and we leave it as an exercise. For instance one can find a closed half-space which contains $F$ and which touches $F$ at the boundary, and then choose $\phi(u)$ on the ray which emanates from that point in the direction orthogonal to the hyperplane and away from $F$.

Thus we have now chosen points $\phi(u) \in \mathbb{R}^{n} \backslash g(F)$ for all $u \in I$. We need to modify them slightly to keep them from getting too close to each other.

Lemma 3.26. For each $u \in I$ we can find a point $\psi(u) \in \mathbb{R}^{n} \backslash g(F)$ with the following properties.

i) If $u \in I_{0}$, then

$$
|\psi(u)-g(\pi(u))| \leq C|g(\pi(u))-g(\rho(u))|
$$

and

$$
\operatorname{dist}(\psi(u), g(F)) \geq C^{-1}|g(\pi(u))-g(\rho(u))| \text {. }
$$


ii) If $u \in I_{1}$, then

$$
\begin{aligned}
C^{-1} \frac{\operatorname{diam} g(F)}{\operatorname{diam} F} \operatorname{dist}(u, F) & \leq \operatorname{dist}(\psi(u), g(F)) \\
& \leq C \frac{\operatorname{diam} g(F)}{\operatorname{diam} F} \operatorname{dist}(u, F) .
\end{aligned}
$$

iii) There is a number $c \in(0,1 / 10)$ such that the balls

$$
\beta(u)=\bar{B}(\psi(u), c \operatorname{dist}(\psi(u), g(F))), \quad u \in I,
$$

have disjoint doubles.

The constants $C$ and $c$ depend only on $n$, the seriousness constant for $F$, and the function which governs the quasisymmetry of $g$.

To prove this we basically want to take the $\psi(u)$ 's to be the same as the $\phi(u)$ 's, but with some small perturbation to get the disjointness condition iii). This will require a small coding argument, and first we need to control some multiplicities.

Sublemma 3.30. For each $u \in I$ there are at most a bounded number of $v \in I$ with

$$
|\phi(v)-\phi(u)| \leq \frac{1}{2} \operatorname{dist}(\phi(u), g(F))
$$

To prove this we need the following.

Claim 3.32. If $u, v \in I$ satisfy (3.31), then

$$
|u-v| \leq C \operatorname{dist}(u, F)
$$

and

$$
C^{-1} \operatorname{dist}(u, F) \leq \operatorname{dist}(v, F) \leq C \operatorname{dist}(u, F)
$$

for a suitable constant $C$.

Let $u, v \in I$ be given, with $u$ and $v$ satisfying (3.31). Notice that (3.31) implies that

$$
\frac{1}{2} \operatorname{dist}(\phi(u), g(F)) \leq \operatorname{dist}(\phi(v), g(F)) \leq \frac{3}{2} \operatorname{dist}(\phi(u), g(F)) .
$$


Suppose first that $u, v \in I_{1}$. In this case we can get (3.34) from (3.35) and (3.25). This implies (3.33) immediately, because dist $(u, F)$ $\geq b \operatorname{diam} F$.

Now suppose that exactly one of $u$ and $v$ lies in $I_{1}$, let us say $v$. From (3.25) we get that

$$
\operatorname{dist}(\phi(v), g(F)) \geq b \operatorname{diam} g(F) .
$$

On the other hand we have that

$$
\operatorname{dist}(\phi(u), g(F)) \leq C \operatorname{diam} g(F)
$$

because of (3.22). Using (3.35) we conclude that

$$
C^{-1} \operatorname{diam} g(F) \leq \operatorname{dist}(\phi(u), g(F))
$$

and

$$
\operatorname{dist}(\phi(v), g(F)) \leq C \operatorname{diam} g(F) \text {. }
$$

Going back to (3.25) we get that

$$
C^{-1} \operatorname{diam} F \leq \operatorname{dist}(v, F) \leq C \operatorname{diam} F .
$$

Let us check that

$$
C^{-1} \operatorname{diam} F \leq \operatorname{dist}(u, F) \leq C \operatorname{diam} F
$$

The upper bound is automatic, because $u \in I_{0}$, the lower bound is the interesting one. It follows from (3.38.a)), Lemma 3.21, and the quasisymmetry of $g$ on $F$.

These last two estimates imply (3.34), and (3.33) follows since $\operatorname{dist}(u, F)$ is bounded from below by a constant times diam $F$. (This would also be true if we switched the roles of $u$ and $v$, and immediately so, since we were assuming that $v \in I_{1}$.)

We are left with the case where both $u$ and $v$ lie in $I_{0}$. Set $R(x)=$ $|g(\pi(x))-g(\rho(x))|$ for $x=u, v$. Then

$$
C^{-1} R(x) \leq \operatorname{dist}(\phi(x), g(F)) \leq C R(x)
$$

when $x=u, v$, because of Lemma 3.21. Thus

$$
C^{-1} R(u) \leq R(v) \leq C R(u)
$$


by (3.35). On the other hand $|\phi(x)-g(\pi(x))| \leq C R(x)$ when $x=u, v$, because of (3.22), and this implies that $|\phi(x)-g(\rho(x))| \leq C R(x)$ for $x=u, v$ too. Using our assumption (3.31) we get that all the points $g(\pi(u)), g(\pi(v)), g(\rho(u))$, and $g(\rho(v))$ have mutual distance bounded by $C R(u)$, and also by $C R(v)$. Quasisymmetry then applies to say that the points $\pi(u), \pi(v), \rho(u), \rho(v)$ all have mutual distances bounded by $C|\pi(u)-\rho(u)|$, and by $C|\pi(v)-\rho(v)|$. In particular

$$
C^{-1}|\pi(v)-\rho(v)| \leq|\pi(u)-\rho(u)| \leq C|\pi(v)-\rho(v)| .
$$

This implies (3.34), because of (3.20). We also get (3.33) from these bounds on the mutual distances and (3.20). This proves Claim 3.32.

Now let us derive Sublemma 3.30 from the claim. Fix $u \in I$, and let $I(u)$ denote the set of $v \in I$ for which (3.31) holds. Thus (3.33) and (3.34) hold for all $v \in I(u)$. Consider the collection of balls $B(v), v \in I(u)$, where $B(v)$ is as in Lemma 2.6. These balls all have approximately the same radius as $B(u)$, because of (3.34), and they are all contained in the ball $k B(u)$, where $k$ is a large constant, because of (3.33) and (3.34). They are also disjoint, because of Lemma 2.6. This implies a bound on their total number, and Sublemma 3.30 follows.

Let us return now to the proof of Lemma 3.26.

Sublemma 3.44. If the constant $c>0$ is chosen small enough, then for each $u \in I$ we can find a point $\psi(u) \in \mathbb{R}^{n} \backslash g(F)$ such that

$$
|\phi(u)-\psi(u)| \leq 10^{-2} \operatorname{dist}(\phi(u), g(F))
$$

and property iii) of Lemma (3.26) holds.

To do this we arrange the points in $I$ as a sequence $\left\{u_{j}\right\}_{j=1}^{\infty}$, in which each element of $I$ appears exactly once, and we choose $\psi\left(u_{j}\right)$ for one $j$ after another. More precisely we want to choose these points so that for each $j$ we have that (3.45) holds for $u=u_{i}, i=1, \ldots, j$, and, if the balls $\beta(u)$ are as defined in Lemma 3.26.iii), then for each $j$ we have that

$$
2 \beta\left(u_{i}\right) \cap 2 \beta\left(u_{k}\right)=\varnothing \quad \text { when } 1 \leq i<k \leq j .
$$

If we can do this for each $j$ then we shall be finished. 
Set $\psi\left(u_{1}\right)=\phi\left(u_{1}\right)$. This satisfies all the requirements for $j=1$ trivially.

Suppose that $\psi\left(u_{i}\right)$ has been chosen for $i<j$ in accordance with the requirements stated above, and let us try to choose $\psi\left(u_{j}\right)$. Of course the disjointness property (3.46) is the thing that we have to keep our eyes on, and it is only an issue for $i<j, k=j$.

Consider first an $i<j$ such that

$$
\left|\phi\left(u_{i}\right)-\phi\left(u_{j}\right)\right| \geq \frac{1}{2} \operatorname{dist}\left(\phi\left(u_{j}\right), g(F)\right)
$$

We are assuming that we chose $\psi\left(u_{i}\right)$ so that (3.45) holds. If we are also careful to choose $\psi\left(u_{j}\right)$ so that (3.45) holds, then (3.47) will ensure that the disjointness property (3.46) will hold (with $k=j$ ) as soon as $c$ is small enough. This is not hard to check, using also a computation like (3.35).

The interesting issue is to deal with the $i$ 's such that (3.47) fails, so that

$$
\left|\phi\left(u_{i}\right)-\phi\left(u_{j}\right)\right|<\frac{1}{2} \operatorname{dist}\left(\phi\left(u_{j}\right), g(F)\right)
$$

The point is that Sublemma 3.30 ensures that there are at most a bounded number of such $i$ 's. If $c$ is chosen small enough then we can choose $\psi\left(u_{j}\right)$ so that (3.45) holds and so that (3.46) holds for these dangerous $i$ 's. This is not hard to see, the point is that we have only to avoid a bounded number of points in a given ball, and we can then get a $c$ which is bounded from below in a way that depends on our bound on the number of bad points. This is slightly vague, but the reader is probably happier filling in the details rather than reading them.

Thus one can choose $\psi\left(u_{j}\right)$ so as to have the required properties. We can repeat this indefinitely to do this for all the $u_{j}$ 's, and Sublemma 3.44 follows from this, as noted above.

Let us now finish the proof of Lemma 3.26. We take $\psi(u)$ to be as provided in Sublemma 3.44, so that we have property iii) of Lemma 3.26 already. There remains the problem of verifying properties i) and ii) of Lemma 3.26. We want to derive them from (3.45) and the corresponding properties of $\phi(u)$. Notice first that (3.45) implies that

$$
\frac{1}{2} \operatorname{dist}(\phi(u), g(F)) \leq \operatorname{dist}(\psi(u), g(F)) \leq 2 \operatorname{dist}(\phi(u), g(F))
$$


as one can easily check. From here we get (3.29) when $u \in I_{1}$, using also the equality (3.25) for $\phi(u)$. Similarly (3.28) holds when $u \in I_{0}$, because of (3.49) and (3.23), while (3.27) follows from (3.22), (3.45), and the fact that

$$
\begin{aligned}
C^{-1}|g(\pi(u))-g(\rho(u))| & \leq \operatorname{dist}(\phi(u), g(F)) \\
& \leq C|g(\pi(u))-g(\rho(u))|
\end{aligned}
$$

(which itself comes from combining (3.22) and (3.23)).

This completes the proof of Lemma 3.26.

Define $h: F \cup I \rightarrow \mathbb{R}^{n}$ by

$$
h= \begin{cases}g & \text { on } F \\ \psi & \text { on } I\end{cases}
$$

We want to show that this mapping is quasisymmetric. This is not difficult but neither is it pleasant. We begin with small observations.

Lemma 3.52. If $p, q \in F \cup I, p \neq q$, then

$$
|p-q| \geq \frac{1}{6}(\operatorname{dist}(p, F)+\operatorname{dist}(q, F)) \text {. }
$$

This follows easily from (2.3).

At the moment $\pi(u)$ and $\rho(u)$ are defined only for $u \in I_{0}$. We extend them to $u \in F$ simply by taking $\pi(u)=\rho(u)=u$ when $u \in F$.

Lemma 3.54. Let $x, z \in F \cup I_{0}$ be given, and suppose that $u$ is either $x, \pi(x)$, or $\rho(x)$, and that $w$ is either $z, \pi(z)$, or $\rho(z)$. Then

$$
|u-w| \leq C|x-z|
$$

where $C$ depends only on the seriousness constant of $F$.

This is an easy consequence of Lemma 3.52 and (3.20).

Lemma 3.56. If $x$ and $z$ are distinct elements of $F \cup I$, then

$$
|h(x)-h(z)| \geq C^{-1}(\operatorname{dist}(h(x), g(F))+\operatorname{dist}(h(z), g(F))),
$$

where $C$ depends only on the dimension n, the seriousness constant of $F$, and the function that governs the quasisymmetry of $g$. 
Indeed, if either $x$ or $z$ lies in $F$ then this is a tautology. If both $x$ and $z$ lie in $I$, then this follows from Lemma 3.26.iii).

Lemma 3.58. Let $x, z \in F \cup I_{0}$ be given, and suppose that $u$ is either $x, \pi(x)$, or $\rho(x)$, and that $w$ is either $z, \pi(z)$, or $\rho(z)$. Then

$$
|h(u)-h(w)| \leq C|h(x)-h(z)|
$$

This constant depends only on the dimension $n$, the seriousness constant of $F$, and the function that governs the quasisymmetry of $g$.

Let us check that

$$
\operatorname{dist}(h(p), g(F)) \leq|h(p)-h(q)| \leq C \operatorname{dist}(h(p), g(F))
$$

when $p \in F \cup I_{0}$ and $q$ is either $\pi(p)$ or $\rho(p)$. This is trivial when $p \in F$, all the relevant quantities vanish, and so we need only consider $p \in I_{0}$. The first inequality follows from the fact that $q \in F$ by definitions. The second inequality follows from (3.27) and (3.28). (Think first about $q=\pi(p)$ and then $q=\rho(p)$. Remember that $h(p)=\psi(p)$, by (3.51).) Thus (3.60) is true.

The bound (3.59) follows now from Lemma 3.56 and (3.60). This proves Lemma 3.58 .

Lemma 3.61. Let $x, z \in F \cup I_{0}$ be given. We can choose $x^{\prime} \in$ $\{\pi(x), \rho(x)\}$ and $z^{\prime} \in\{\pi(z), \rho(z)\}$ so that

$$
\left|x^{\prime}-z^{\prime}\right| \geq \frac{|x-z|}{16} .
$$

Note that the reverse inequality is provided by Lemma 3.54.

For the proof we follow a suggestion from the Unknown Finn. Let us check first that if $u \in F \cup I_{0}$ and $v \in \mathbb{R}^{n}$ is arbitrary, then

$$
|u-v| \leq 2(|\pi(u)-v|+|\rho(u)-v|) .
$$

We have that

$$
|\pi(u)-v|+|\rho(u)-v| \geq|\pi(u)-\rho(u)| \geq \operatorname{dist}(u, F),
$$


by (3.20). If $|u-v| \leq 2 \operatorname{dist}(u, F)$ then we get (3.63) from (3.64). If $|u-v|>2 \operatorname{dist}(u, F)$ then

$$
\begin{aligned}
|u-v| & \leq|u-\pi(u)|+|\pi(u)-v| \\
& =\operatorname{dist}(u, F)+|\pi(u)-v| \\
& <|u-v| / 2+|\pi(u)-v|,
\end{aligned}
$$

and so $|u-v|<2|\pi(u)-v|$. Thus (3.63) holds in this case too.

From (3.63) (applied twice) we conclude that if $u, v \in F \cup I_{0}$, then

$$
\begin{aligned}
|u-v| \leq & 4(|\pi(u)-\pi(v)|+|\rho(u)-\rho(v)| \\
& +|\pi(u)-\rho(v)|+|\rho(u)-\pi(v)|) .
\end{aligned}
$$

Lemma 3.61 follows from this.

Lemma 3.66. Let $x, y, z \in F \cup I_{0}$ and $t>0$ be given, with $x \neq y$ and $|x-y| \leq t|x-z|$. Then

$$
\operatorname{dist}(h(x), g(F)) \leq C \eta(C t)|h(x)-h(z)|,
$$

where $\eta$ is the function that governs the quasisymmetry of $g$, and where $C$ depends only on $n$, the seriousness constant of $F$, and $\eta$.

This lemma is trivial when $x \in F$, and so we assume that $x \in I_{0}$. Lemma 3.52 permits us to convert our hypothesis into

$$
\operatorname{dist}(x, F) \leq 6 t|x-z| \text {. }
$$

Let $x^{\prime}, z^{\prime}$ be as in Lemma 3.61. We can convert (3.68) into

$$
\left|x^{\prime}-q\right| \leq C t\left|x^{\prime}-z^{\prime}\right|
$$

for $q=\pi(x), \rho(x)$. This follows from (3.68), using (3.62) and (3.20). All these points $x^{\prime}, z^{\prime}, q$ lie in $F$, on which $h$ equals $g$, and so we can use the quasisymmetry of $g$ to get

$$
\left|h\left(x^{\prime}\right)-h(q)\right| \leq \eta(C t)\left|h\left(x^{\prime}\right)-h\left(z^{\prime}\right)\right|
$$

for $q=\pi(x), \rho(x)$, where $\eta$ is the function that governs the quasisymmetry of $g$. Because $x^{\prime}$ is one of $\pi(x), \rho(x)$, we can take $q$ to be the other one, and we get

$$
|h(\pi(x))-h(\rho(x))| \leq \eta(C t)\left|h\left(x^{\prime}\right)-h\left(z^{\prime}\right)\right| .
$$


Lemma 3.26.i) permits us to replace this with

$$
\operatorname{dist}(h(x), g(F)) \leq C \eta(C t)\left|h\left(x^{\prime}\right)-h\left(z^{\prime}\right)\right|
$$

Using Lemma 3.58 we get that

$$
\operatorname{dist}(h(x), g(F)) \leq C \eta(C t)|h(x)-h(z)| \text {. }
$$

This proves Lemma 3.66.

Lemma 3.74. The restriction of $h$ to $F \cup I_{0}$ is quasisymmetric, with the quasisymmetry governed by the function $C \eta(C t)$, where $\eta$ is the function that governs the quasisymmetry of $g$, and where $C$ depends only on the dimension $n$, the seriousness constant of $F$, and $\eta$.

Let $x, y, z \in F \cup I_{0}, t>0$, be given, such that

$$
|x-y| \leq t|x-z|
$$

We want to show that

$$
|h(x)-h(y)| \leq C \eta(C t)|h(x)-h(z)|
$$

where $C$ and $\eta$ are as above. We may as well assume that $y \neq x$.

Let $x^{\prime}, z^{\prime}$ be associated to $x, z$ as in Lemma 3.61. Then (3.75) implies that $|x-y| \leq C t\left|x^{\prime}-z^{\prime}\right|$, by (3.62). Therefore

$$
\left|x^{\prime}-q\right| \leq C t\left|x^{\prime}-z^{\prime}\right|
$$

for each of $q=\pi(y), \rho(y)$, because of Lemma 3.54 (applied to $x$ and $y$ ). Because $x^{\prime}, z^{\prime}, \pi(y), \rho(y)$ all lie in $F$, and because $h$ equals $g$ on $F$, we conclude that

$$
\left|h\left(x^{\prime}\right)-h(q)\right| \leq \eta(C t)\left|h\left(x^{\prime}\right)-h\left(z^{\prime}\right)\right|
$$

for each of $q=\pi(y), \rho(y)$, where $\eta$ is the function that governs the quasisymmetry of $g$. Lemma 3.58 permits us to convert this into

$$
\left|h\left(x^{\prime}\right)-h(q)\right| \leq C \eta(C t)|h(x)-h(z)|,
$$

for each of $q=\pi(y), \rho(y)$. 
Lemma 3.66 implies that

$$
\operatorname{dist}(h(x), g(F)) \leq C \eta(C t)|h(x)-h(z)| .
$$

From (3.60) (with $p=x, q=x^{\prime}$ ) we have that $\left|h(x)-h\left(x^{\prime}\right)\right| \leq$ $C$ dist $(h(x), g(F))$. Combining these estimates with (3.79) we get that

$$
|h(x)-h(q)| \leq C \eta(C t)|h(x)-h(z)|,
$$

for each of $q=\pi(y), \rho(y)$.

In particular we have that

$$
|h(\pi(y))-h(\rho(y))| \leq C \eta(C t)|h(x)-h(z)|,
$$

and hence

$$
|h(y)-h(\pi(y))| \leq C \eta(C t)|h(x)-h(z)|
$$

by (3.27). Combining this with (3.81) (with $q=\pi(y)$ ) we get that

$$
|h(x)-h(y)| \leq C \eta(C t)|h(x)-h(z)| .
$$

This proves the lemma.

Our next main goal is to prove the following.

Lemma 3.85. $h: F \cup I \rightarrow \mathbb{R}^{n}$ is quasisymmetric, with bounds that depend only on the dimension $n$, the seriousness constant of $F$, and the function that governs the quasisymmetry of $g$.

In order to prove this we may as well assume that

$$
\operatorname{diam} F=\operatorname{diam} g(F)=1 \text {, }
$$

because we can always make rescalings on the domain and image without altering our assumptions. This assumption will be in force throughout the proof of Lemma 3.85.

In the following the constants $C$ are permitted to depend only on the dimension $n$, the seriousness constant of $F$, and the function that governs the quasisymmetry of $g$.

The reader might wish to review the definitions of $I_{0}$ and $I_{1}$, which are given shortly before (3.20). In particular they imply that

$$
\operatorname{diam}\left(F \cup I_{0}\right) \leq 3 .
$$


Using this and Lemma 3.26.i) we get that

$$
\operatorname{diam} h\left(F \cup I_{0}\right) \leq C
$$

Sublemma 3.89. If $p \in F \cup I$ and $q \in I_{1}$, then

$$
C^{-1}|p-q| \leq|h(p)-h(q)| \leq C|p-q|,
$$

We may as well assume that $p \neq q$.

Let us prove the upper bound first. We can do it crudely, starting with

$$
\begin{aligned}
|h(p)-h(q)| \leq & \operatorname{dist}(h(p), g(F))+\operatorname{diam} g(F) \\
& +\operatorname{dist}(h(q), g(F)) \\
= & \operatorname{dist}(h(p), g(F))+1+\operatorname{dist}(h(q), g(F)) .
\end{aligned}
$$

On the other hand we have that

$$
\begin{aligned}
|p-q| & \geq \frac{1}{6}(\operatorname{dist}(p, F)+\operatorname{dist}(q, F)) \\
& \geq C^{-1}(\operatorname{dist}(p, F)+\operatorname{dist}(q, F)+1) .
\end{aligned}
$$

The first inequality comes from Lemma 3.52, while the second follows from our assumption that $q \in I_{1}$. From (3.29) we get that

$$
\operatorname{dist}(h(q), g(F)) \leq C \operatorname{dist}(q, F) \text {. }
$$

If $p \in I_{1}$ we have the analogous inequality for $p$ instead of $q$, and then the upper bound in (3.90) follows from (3.91) and (3.92). If $p \in F \cup I_{0}$, then dist $(h(p), g(F)) \leq C$ by (3.88), and the upper bound in (3.90) again follows from (3.91) and (3.92). This proves the upper bound in (3.90).

Let us now prove the lower bound. Lemma 3.56 implies that

$$
|h(p)-h(q)| \geq C^{-1}(\operatorname{dist}(h(p), g(F))+\operatorname{dist}(h(q), g(F))),
$$

Using (3.29) we get that

$$
\operatorname{dist}(h(q), g(F)) \geq C^{-1} \operatorname{dist}(q, F) \geq C^{-1}(\operatorname{dist}(q, F)+1) .
$$


We are also employing the assumption that $q \in I_{1}$ to get the last inequality. If $p \in I_{1}$, then we get the analogue of (3.95) for $p$ as well, and then

$$
|h(p)-h(q)| \geq C^{-1}(\operatorname{dist}(q, F)+1+\operatorname{dist}(p, F)) .
$$

This implies the lower bound in (3.90), using also (3.86). If $p \in F \cup I_{0}$, then we have

$$
\begin{aligned}
|h(p)-h(q)| & \geq C^{-1}(\operatorname{dist}(q, F)+1) \\
& \geq C^{-1}\left(\operatorname{dist}(q, F)+\operatorname{diam}\left(F \cup I_{0}\right)\right),
\end{aligned}
$$

by (3.87). This implies the lower bound in (3.90) in this case.

This proves Sublemma 3.89.

Let us come back now to the proof of Lemma 3.85. Let $x, y, z \in$ $F \cup I$ and $t>0$ be given, with

$$
|x-y| \leq t|x-z|
$$

We want to show that

$$
|h(x)-h(y)| \leq \theta(t)|h(x)-h(z)|,
$$

where $\theta:[0, \infty) \rightarrow[0, \infty)$ vanishes at the origin, is continuous at the origin, and is bounded on bounded sets. (Lemma 2.42 is relevant here.)

If all three of $x, y, z$ lie in $F \cup I_{0}$ then we can use Lemma 3.74 to get the required estimate.

If $x \in I_{1}$ then we have that

$$
|h(x)-h(y)| \leq C t|h(x)-h(z)|
$$

because of Sublemma 3.89. Thus we may assume that

$$
x \in F \cup I_{0}
$$

If both $y$ and $z$ lie in $I_{1}$, then we get (3.100) again from Sublemma 3.89. If they both lie in $F \cup I_{0}$ then all three points lie there and we are back to a case that we know. Thus we may require that

$$
\text { exactly one of } y \text { and } z \text { lies in } F \cup I_{0} \text {. }
$$


Let us pause for a small observation.

Sublemma 3.103. If $p \in F \cup I_{0}$ and $q \in I_{1}$, then $|p-q| \geq C^{-1}$.

Indeed, in this case $6|p-q| \geq \operatorname{dist}(q, F)$, by Lemma 3.52, and Sublemma 3.103 follows from the assumption that $q \in I_{1}$.

Let us come back now to the task of proving an estimate like (3.99) under the conditions (3.98), (3.101), and (3.102). Assume first that $y \in I_{1}$. In this case we have

$$
|x-y| \geq C^{-1}
$$

by Sublemma 3.103. This implies that $\operatorname{diam} F \leq C t|x-z|$, and so the quasisymmetry of $h$ on $F \cup I_{0}$ (Lemma 3.74) implies that

$$
1=\operatorname{diam} g(F) \leq C \eta(C t)|h(x)-h(z)|,
$$

where $\eta$ is the function that controls the quasisymmetry of $g$. On the other hand

$$
|h(x)-h(y)| \leq C|x-y|
$$

by Sublemma 3.89, and so

$$
\begin{aligned}
|h(x)-h(y)| & \leq C t|x-z| \\
& \leq C t \operatorname{diam}\left(F \cup I_{0}\right) \\
& \leq C t \leq C t \eta(C t)|h(x)-h(z)|
\end{aligned}
$$

by (3.87) and (3.105). This is the kind of estimate that we want.

Assume now that $z \in I_{1}$, so that $x, y \in F \cup I_{0}$. Notice that

$$
|x-z| \geq C^{-1}
$$

and

$$
C^{-1}|x-z| \leq|h(x)-h(z)| \leq C|x-z|
$$

by Sublemmas 3.103 and 3.89. Our assumption (3.98) implies that either

$$
|x-y| \leq \sqrt{t}
$$


or

$$
1 \leq \sqrt{t}|x-z|
$$

Assume first that (3.111) holds. Then we have that

$$
\begin{aligned}
|h(x)-h(y)| & \leq \operatorname{diam} h\left(F \cup I_{0}\right) \\
& \leq C \\
& \leq C \sqrt{t}|x-z| \\
& \leq C \sqrt{t}|h(x)-h(z)|,
\end{aligned}
$$

by (3.109). This estimate does the job for this case. So suppose now that (3.110) holds. In this case we have that

$$
|h(x)-h(y)| \leq \omega(\sqrt{t})
$$

for a certain function $\omega$ on $[0, \infty)$ which vanishes at the origin, is continuous at the origin, and is bounded. Indeed, we have $x, y \in F \cup I_{0}$ in the present situation, and so (3.113) follows from the quasisymmetry of $h$ on $F \cup I_{0}$ and (3.87), (3.88). Using (3.108) and (3.109) we get that

$$
|h(x)-h(y)| \leq C \omega(\sqrt{t})|h(x)-h(z)|,
$$

which does the job in this case.

This completes the proof of Lemma 3.85.

Note that we have not tried to give sharp estimates here, it was more interesting to just get it over with.

Let us now finish the proof of Proposition 1.14. Let $S$ be as in (3.1), and let us define a mapping $G$ on $S$. We set $G=g$ on $F$, and if $x \in I$ we set

$$
G(p)=h(x)+\frac{c \operatorname{dist}(h(x), g(F))}{20^{-1} \operatorname{dist}(x, F)}(p-x) \quad \text { for } p \in B(x)
$$

Here $c$ is chosen as in Lemma 3.26.iii); the ratio in (3.115) is simply the ratio between the radius of the ball $\beta(x)$ defined in Lemma 3.26.iii) and the radius of the ball $B(x)$ which is used in (3.1). In fact $G$ maps the center of $B(x)$ to the center $\psi(x)=h(x)$ of $\beta(x)$, by definitions, and so we get that $G(B(x))=\beta(x)$ for all $x \in I$. 
We want to say that $G: S \rightarrow \mathbb{R}^{n}$ is quasisymmetric with a suitable bound. We apply Lemma 2.29 , with $A=F, H=G$, and with the balls $B_{i}$ and $\beta_{i}$ taken to be the $B(x)^{\text {'s }}$ and $\beta(x)$ 's, with the obvious changes in notation. We have to check that the hypotheses of Lemma 2.29 hold in this case. The requirement that "the restriction of $H$ to $A^{\prime}$ is quasisymmetric" is satisfied in this case because of Lemma 3.85. We just checked that $H$ maps the $B_{i}$ 's onto the $\beta_{i}$ 's, and the restriction of $H$ to each $B_{i}$ is a similarity, and hence quasisymmetric with uniform bounds. We know from Lemma 2.6 that the doubles of the $B_{i}$ 's are disjoint, and they are disjoint from $A=F$ by their definition. Similarly the $\beta_{i}$ 's have disjoint doubles because of Lemma 3.26.iii), and the doubles are disjoint from $H(A)=g(F)$ by their definition. The bounds (2.30) also follow from the definitions of the $B_{i}$ 's and $\beta_{i}$ 's. Thus the hypotheses of Lemma 2.29 are satisfied in this case, and we conclude that $H: A^{*} \rightarrow \mathbb{R}^{n}$ is quasisymmetric, which is the same as saying that $G: S \rightarrow \mathbb{R}^{n}$ is quasisymmetric. Of course we also get the correct bounds.

This completes the proof of Proposition 1.14.

\section{The proof of Proposition 1.15.}

Let us address first a preliminary point.

Proposition 4.1. If $F$ is a serious subset of $\mathbb{R}^{n}$ and $g: F \rightarrow \mathbb{R}^{n}$ is quasisymmetric, then $g(F)$ is also serious, with a constant which depends only on the seriousness constant of $F$ and the function that governs the quasisymmetry of $g$.

This is less amusing than Proposition 1.15, because it is really a fact about (quasi-) metric spaces rather than subsets of Euclidean spaces.

Let $x \in F$ be given. For each $0<t<\operatorname{diam} F$ choose a point $y(t) \in F$ so that

$$
C_{0}^{-1} t \leq|x-y(t)| \leq t
$$

where $C_{0}$ is the seriousness constant of $F$.

Claim 4.3. There is a constant $C>0$ so that for each $0<s<$ $\operatorname{diam} g(F)$ we can find $a 0<t<\operatorname{diam} F$ such that

$$
C^{-1} s \leq|g(x)-g(y(t))| \leq C s .
$$


To prove the claim we use a continuity argument. We have that

$$
C^{-1} \operatorname{diam} g(F) \leq|g(x)-g(y(t))| \leq C \operatorname{diam} g(F)
$$

when $t>\operatorname{diam}(F) / 2$ and $\operatorname{diam} F<\infty$, because of (4.2) and quasisymmetry, and

$$
\lim _{t \rightarrow \infty}|g(x)-g(y(t))|=\infty
$$

when $\operatorname{diam} F=\infty$. The continuity of $g$ implies that

$$
\lim _{t \rightarrow 0}|g(x)-g(y(t))|=0 .
$$

We also have that

$$
C^{-1}|g(x)-g(y(t))| \leq|g(x)-g(y(t / 2))| \leq C|g(x)-g(y(t))|
$$

when $0<t<\operatorname{diam} F$, by quasisymmetry. The claim follows from these three observations.

Proposition 4.1 follows easily from Claim 4.2.

Now let us prove Proposition 1.15.

Let $S$ be a strong subset of $\mathbb{R}^{n}$ and let $G: S \rightarrow \mathbb{R}^{n}$ be quasisymmetric. We want to show that $G(S)$ is strong, with bounds. We know from Proposition 4.1 that $G(S)$ is serious. Of course $S$ is unbounded, since it is strong, and so $G(S)$ is also unbounded. $G(S)$ is also closed, since $S$ is.

Let $x \in \mathbb{R}^{n} \backslash G(S)$ be given. Choose $x_{0} \in G(S)$ so that $\operatorname{dist}(x, G(S))$ $=\left|x-x_{0}\right|$, and choose $x_{1} \in G(S)$ so that

$$
C^{-1}\left|x_{1}-x_{0}\right| \leq\left|x-x_{0}\right| \leq\left|x_{1}-x_{0}\right| \text {. }
$$

We can do this because $G(S)$ is serious and unbounded.

We can apply Lemma 3.17 (with $X=G(S), f=G^{-1}$ (remember Lemma 1.4), $x=x_{0}, y=x_{1}$, and $\left.z=x\right)$ to get a point $w \in \mathbb{R}^{n} \backslash S$ such that

$$
\left|w-G^{-1}\left(x_{0}\right)\right| \leq C\left|G^{-1}\left(x_{0}\right)-G^{-1}\left(x_{1}\right)\right|
$$

and

$$
\operatorname{dist}(w, S) \geq C^{-1}\left|G^{-1}\left(x_{0}\right)-G^{-1}\left(x_{1}\right)\right|
$$


Our assumption that $S$ is strong implies the existence of a point $v \in S$ such that

$$
|w-v| \leq C \operatorname{dist}(w, S)
$$

and

$$
\operatorname{dist}\left(v, \mathbb{R}^{n} \backslash S\right) \geq C^{-1} \operatorname{dist}(w, S) .
$$

Let us rephrase (4.13) as

$$
B\left(v, C^{-1} \operatorname{dist}(w, S)\right) \subseteq S .
$$

Set $y=G(v)$. We want to show that

$$
|x-y| \leq C \operatorname{dist}(x, G(S))
$$

and

$$
\operatorname{dist}\left(y, \mathbb{R}^{n} \backslash G(S)\right) \geq C^{-1} \operatorname{dist}(x, G(S)) .
$$

We shall derive these from (4.12) and (4.13) using the quasisymmetry of $G$.

From (4.10) and (4.11) we have that

$$
\begin{aligned}
C^{-1}\left|G^{-1}\left(x_{0}\right)-G^{-1}\left(x_{1}\right)\right| & \leq \operatorname{dist}(w, S) \\
& \leq C\left|G^{-1}\left(x_{0}\right)-G^{-1}\left(x_{1}\right)\right| .
\end{aligned}
$$

Combining (4.12) and (4.10) we get that

$$
\left|v-G^{-1}\left(x_{0}\right)\right| \leq C\left|G^{-1}\left(x_{0}\right)-G^{-1}\left(x_{1}\right)\right| .
$$

Since $G$ is quasisymmetric we conclude that

$$
\left|y-x_{0}\right| \leq C\left|x_{0}-x_{1}\right|
$$

Using (4.9) we can convert this into

$$
|y-x| \leq C\left|x-x_{0}\right|
$$

This implies (4.15), because of our choice of $x_{0}$. 
It remains to prove (4.16), which we can rewrite as

$$
B\left(y, C^{-1} \operatorname{dist}(x, G(S))\right) \subseteq G(S) .
$$

Of course the point is to use (4.13). Let $B$ denote the ball on the left side of (4.14). Because of invariance of domain we have that $G(B)$ is an open subset of $\mathbb{R}^{n}$ which contains $y$.

Claim 4.22. $\operatorname{dist}(y, G(B \backslash(B / 2))) \geq C^{-1} \operatorname{dist}(x, G(S))$.

To see this we want to show that

$$
\operatorname{dist}(y, G(B \backslash(B / 2))) \geq C^{-1}\left|x_{0}-x_{1}\right| \text {. }
$$

To prove this we use the quasisymmetry of $G$. Let $z \in B \backslash(B / 2)$ be given. Then

$$
C^{-1}\left|G^{-1}\left(x_{0}\right)-G^{-1}\left(x_{1}\right)\right| \leq|z-v| \leq C\left|G^{-1}\left(x_{0}\right)-G^{-1}\left(x_{1}\right)\right|
$$

because of (4.17). This implies that

$$
|z-v| \geq C^{-1}\left|v-G^{-1}\left(x_{i}\right)\right|, \quad i=0,1,
$$

by (4.18). Using this and quasisymmetry it is not hard to show that

$$
|G(z)-y| \geq C^{-1}\left|x_{0}-x_{1}\right|
$$

(Remember that $y=G(v)$.) With (4.26) in hand we get (4.23) immediately, and Claim 4.22 follows from (4.9) and our choice of $x_{0}$.

Let us now use the claim to derive (4.21). Let $p \in \mathbb{R}^{n} \backslash G(B)$ be chosen so that $|p-y|$ is as small as possible. We can do this because $G(B)$ is an open subset of $\mathbb{R}^{n}$, and we also get that $|p-y|>0$. Set $p_{t}=$ $y+t(p-y)$ for $0<t<1$, so that each $p_{t}$ lies in $G(B)$. For $t$ sufficiently close to 1 we must have that $p_{t} \in G(B \backslash(B / 2))$; for if this were not the case, then $p$ would lie in $G(\bar{B} / 2)$, in contradiction to our choice of $p$ (lying outside $G(B))$. Thus $p_{t} \in G(B \backslash(B / 2))$ for $t$ sufficiently close to 1 , and we conclude from Claim 4.22 that $|p-y| \geq C^{-1} \operatorname{dist}(x, G(S))$. This proves (4.16).

Thus we have proved that $G(S)$ is a strong set, and Proposition 1.15 follows. 


\section{The proof of Proposition 1.16.}

Let $S$ be a strong subset of $\mathbb{R}^{n}$, and let us try to prove (1.17). It suffices to show that there is a constant $k>1$ so that

$$
|S \cap B(p, k r)| \geq k^{-1}|B(p, r) \backslash S|
$$

for all $p \in S$ and $r>0$.

Let $p \in S$ and $r>0$ be given, and let us apply Lemma 2.1 with $E=S$ and $H=B(p, r) \backslash S$. Lemma 2.1 produces a subset $I$ of $H$ with the properties listed there. From (2.2) we get that

$$
|B(p, r) \backslash S| \leq C \sum_{x \in I}(\operatorname{dist}(x, S))^{n} .
$$

Given $x \in I$ choose $\tau(x) \in S$ so that

$$
|x-\tau(x)| \leq C \operatorname{dist}(x, S)
$$

and

$$
\operatorname{dist}\left(\tau(x), \mathbb{R}^{n} \backslash S\right) \geq C^{-1} \operatorname{dist}(x, S) .
$$

We can do this because $S$ is strong. Note that these inequalities imply that

$$
C^{-1} \operatorname{dist}(x, S) \leq \operatorname{dist}\left(\tau(x), \mathbb{R}^{n} \backslash S\right) \leq C \operatorname{dist}(x, S) .
$$

Given $x \in I$, set

$$
\beta(x)=B\left(\tau(x), \operatorname{dist}\left(\tau(x), \mathbb{R}^{n} \backslash S\right) / 2\right) .
$$

From (5.2) and (5.5) we have that

$$
|B(p, r) \backslash S| \leq C \sum_{x \in I}|\beta(x)| .
$$

We want to use this to prove (5.1).

Lemma 5.8. For each $x \in I$ there are at most a bounded number of $z \in I$ such that $\beta(x)$ intersects $\beta(z)$. 
Suppose that $x, z \in I$ satisfy $\beta(x) \cap \beta(z) \neq \varnothing$. Then

$$
|\tau(x)-\tau(z)|<\frac{1}{2}\left(\operatorname{dist}\left(\tau(x), \mathbb{R}^{n} \backslash S\right)+\operatorname{dist}\left(\tau(z), \mathbb{R}^{n} \backslash S\right)\right) .
$$

This implies that

(5.10) $\frac{1}{3} \operatorname{dist}\left(\tau(x), \mathbb{R}^{n} \backslash S\right) \leq \operatorname{dist}\left(\tau(z), \mathbb{R}^{n} \backslash S\right) \leq 3 \operatorname{dist}\left(\tau(x), \mathbb{R}^{n} \backslash S\right)$.

Using (5.5) we conclude that

$$
C^{-1} \operatorname{dist}(x, S) \leq \operatorname{dist}(z, S) \leq C \operatorname{dist}(x, S) .
$$

We also get that

$$
|x-z| \leq C \operatorname{dist}(x, S)
$$

because of (5.3), (5.9), (5.5), and (5.11).

Let $I(x)$ denote the set of $z \in I$ such that $\beta(x) \cap \beta(z) \neq \varnothing$. From (2.3) and (5.11) we obtain that

$$
|y-z| \geq C^{-1} \operatorname{dist}(x, S) \quad \text { when } y, z \in I(x), y \neq z .
$$

It is easy to see that $I(x)$ can have only a bounded number of elements, using (5.12) and (5.13). This proves the lemma.

Lemma 5.8 permits us to convert (5.7) into

$$
|B(p, r) \backslash S| \leq C\left|\bigcup_{x \in I} \beta(x)\right| \cdot
$$

Let us check that

$$
\bigcup_{x \in I} \beta(x) \subseteq B(p, C r) \cap S
$$

We have $\beta(x) \subseteq S$ from the definition (5.6). We also know that its radius is bounded by $C$ dist $(x, S)$, and this is at most $C|x-p| \leq C r$ for $x \in I$. The inclusion (5.15) follows easily from these observations, and the fact that $I \subseteq B(p, r)$ by definitions.

Combining (5.15) with (5.14) we get (5.1). This completes the proof of Proposition 1.16. 


\section{The proof of Proposition 1.22.}

The proof of Proposition 1.22 is a straightforward consequence of the previous results and the definitions, but let us be slightly careful. Let $S$ be a strong subset of $\mathbb{R}^{n}$ and let $G: S \rightarrow \mathbb{R}^{n}$ be quasisymmetric, as in the proposition. Define the measure $\nu$ on $\mathbb{R}^{n}$ by $\nu(A)=|A \cap G(S)|$. Note that $G(S)$ is a strong subset of $\mathbb{R}^{n}$, because of Proposition 1.15. Thus

$$
C^{-1} r^{n} \leq \nu(B(x, r)) \leq C r^{n}
$$

for some constant $C$ and all $x \in G(S), r>0$, by Proposition 1.16. Of course $\nu$ has support equal to $G(S)$.

Define the measure $\mu$ on $\mathbb{R}^{n}$ by $\mu(A)=|G(A \cap S)|$, as in the statement of Proposition 1.22. Thus $\mu$ is a measure with support equal to $S$ which is obtained by pulling back $\nu$ using the homeomorphism $G$.

That $\mu$ is doubling on $S$, as in Definition 1.18 a), is easy to check, using (6.1) and the quasisymmetry of $G$. The point is that if we are given $x \in S$ and $r>0$, then we can find a ball $B=B(G(x), t)$ such that $G(B(x, r) \cap S) \supseteq B \cap G(S)$ and $G(B(x, 2 r) \cap S) \subseteq k B \cap G(S)$, where $k$ is a constant that does not depend on $x$ or $r$.

To see that $\mu$ is a metric doubling measure on $S$, as in Definition 1.18.b), it suffices to show that

$$
C^{-1}|G(x)-G(y)| \leq \delta(x, y) \leq C|G(x)-G(y)|
$$

for some $C$ and all $x, y \in S$, where $\delta(x, y)$ is as in (1.20). This is sufficient because $d(x, y)=|G(x)-G(y)|$ is obviously a metric on $S$. To get these bounds the main point is that

$$
\begin{aligned}
B\left(G(x), C^{-1}\right. & |G(x)-G(y)|) \cap G(S) \\
& \subseteq G(B(x,|x-y|) \cap S) \\
& \subseteq B(G(x), C|G(x)-G(y)|) \cap G(S) .
\end{aligned}
$$

These inclusions follow from the quasisymmetry of $G$. Once we have them (6.2) follows easily from the definition (1.20) of $\delta(x, y)$ and the estimate (6.1).

This completes the proof of Proposition 1.22. 


\section{The proof of Proposition 1.23.}

Let $S$ be a strong subset of $\mathbb{R}^{n}$, and let $\mu$ be a metric doubling measure on $S$. We want to find a metric doubling measure $\nu$ on all of $\mathbb{R}^{n}$ which equals $\mu$ on $S$.

Let $\left\{Q_{i}\right\}_{i \in I}$ be a Whitney decomposition of $\mathbb{R}^{n} \backslash S$. Thus the $Q_{i}$ 's are closed cubes with disjoint interiors whose union is all of $\mathbb{R}^{n} \backslash S$ and which satisfy

$$
\operatorname{diam} Q_{i} \leq \operatorname{dist}\left(Q_{i}, S\right) \leq 4 \operatorname{diam} Q_{i}
$$

as in [St, Theorem 1, p. 167].

We shall use this Whitney decomposition to define $\nu$, we shall define it in a simple way on each $Q_{i}$ and then combine the pieces. In order to define $\nu$ on the $Q_{i}$ 's we need to look at $\mu$ inside $S$, and we need to use our assumption that $S$ is a strong set.

For each $i \in I$ choose $q_{i} \in Q_{i}$ so that

$$
\operatorname{dist}\left(q_{i}, S\right)=\operatorname{dist}\left(Q_{i}, S\right)
$$

Using the fact that $S$ is a strong set we can find a cousin for each $q_{i}$ inside $S$, namely a point $p_{i}$ such that

$$
\left|p_{i}-q_{i}\right| \leq C \operatorname{dist}\left(q_{i}, S\right)
$$

and

$$
\operatorname{dist}\left(p_{i}, \mathbb{R}^{n} \backslash S\right) \geq C^{-1} \operatorname{dist}\left(q_{i}, S\right) .
$$

These inequalities imply easily that

$$
C^{-1} \operatorname{dist}\left(q_{i}, S\right) \leq \operatorname{dist}\left(p_{i}, \mathbb{R}^{n} \backslash S\right) \leq C \operatorname{dist}\left(q_{i}, S\right) .
$$

Given $i \in I$ set

$$
\beta_{i}=B\left(p_{i}, \operatorname{dist}\left(p_{i}, \mathbb{R}^{n} \backslash S\right) / 2\right) .
$$

Define $\nu$ by

$$
\nu(A)=\mu(A \cap S)+\sum_{i \in I} \frac{\mu\left(\beta_{i}\right)}{\left|Q_{i}\right|}\left|A \cap Q_{i}\right| .
$$


We want to show that this is a metric doubling measure on $\mathbb{R}^{n}$.

(It is not hard to see that (7.7) is the right way to define $\nu$. There are various ways to package this extension, but basically there is only one reasonable way to do it, and this is it.)

The proof that $\nu$ is a metric doubling measure is pretty straightforward, a matter of checking that certain things follow from certain other things. We begin with some small technical observations. The constants $C$ that appear below are allowed to depend only on the dimension $n$, the metric doubling constants for $\mu$, and the strongness constant for $S$.

Lemma 7.8. If $Q_{i}$ is a Whitney cube and $\operatorname{dist}\left(x, Q_{i}\right) \leq \operatorname{diam}\left(Q_{i}\right) / 10$, then

$$
\frac{9}{10} \operatorname{diam} Q_{i} \leq \operatorname{dist}(x, S) \leq 6 \operatorname{diam} Q_{i}
$$

This is an immediate consequence of (7.1).

Lemma 7.10. If two Whitney cubes $Q_{i}$ and $Q_{j}$ satisfy $\operatorname{dist}\left(Q_{i}, Q_{j}\right) \leq$ $\operatorname{diam}\left(Q_{i}\right) / 10$, then

$$
\frac{9}{60} \operatorname{diam} Q_{i} \leq \operatorname{diam} Q_{j} \leq 6 \operatorname{diam} Q_{i}
$$

This follows from (7.1) and Lemma 7.8.

Lemma 7.12. If two Whitney cubes $Q_{i}$ and $Q_{j}$ satisfy $\operatorname{dist}\left(Q_{i}, Q_{j}\right) \leq$ $\operatorname{diam}\left(Q_{i}\right) / 10$, then

$$
C^{-1} \mu\left(\beta_{i}\right) \leq \mu\left(\beta_{j}\right) \leq C \mu\left(\beta_{i}\right) .
$$

If $Q_{i}$ and $Q_{j}$ are as above, then

$$
\left|p_{i}-p_{j}\right| \leq C \operatorname{diam} Q_{i}
$$

by (7.3), (7.2), and (7.11). Also the radii of both $\beta_{i}$ and $\beta_{j}$ are comparable to diam $Q_{i}$, because of (7.5) and (7.2). Thus we conclude that $\beta_{i}$ is contained in some bounded multiple of $\beta_{j}$, and vice-versa. The doubling condition then yields (7.13). 
Lemmma 7.15. For each $i \in I$ there is only a bounded number of $j \in I$ such that $\beta_{i}$ intersects $\beta_{j}$.

This is very similar to Lemma 5.8. If $\beta_{i} \cap \beta_{j} \neq \varnothing$, then one can show that the radii of $\beta_{i}$ and $\beta_{j}$ are the same to within a factor of 3 , for the same reason as in (5.10). This implies that

$$
C^{-1} \operatorname{diam} Q_{i} \leq \operatorname{diam} Q_{j} \leq C \operatorname{diam} Q_{i}
$$

for some constant $C$. Next $\beta_{i} \cap \beta_{j} \neq \varnothing$ implies that $\left|p_{i}-p_{j}\right| \leq$ $C$ diam $Q_{i}$, because of (7.5), (7.2), and (7.1). Using (7.3) we get that

$$
\operatorname{dist}\left(Q_{i}, Q_{j}\right) \leq C \operatorname{diam} Q_{i}
$$

If we fix $i$, then there can be only a bounded number of $j$ 's for which (7.16) and (7.17) are valid, because the $Q_{j}$ 's have disjoint interiors. Lemma 7.15 follows from this.

Lemma 7.18. $\mu(B(x, r)) \leq \nu(B(x, r)) \leq C \mu(B(x, r))$ whenever $x \in S$ and $r>0$.

Let $x \in S$ and $r>0$ be given. The first inequality is trivial. For the second it suffices to show that

$$
\nu(B(x, r) \backslash S) \leq k \mu(B(x, k r))
$$

for some constant $k$, since $\mu$ is doubling on $S$.

Set $J=\left\{i \in I: Q_{i} \cap B(x, r) \neq \varnothing\right\}$. Then

$$
\nu(B(x, r) \backslash S) \leq \sum_{i \in J} \mu\left(\beta_{i}\right)
$$

by the definition (7.7) of $\nu$. Lemma 7.15 permits us to convert this into

$$
\nu(B(x, r) \backslash S) \leq C \mu\left(\bigcup_{i \in J} \beta_{i}\right)
$$

Thus we are reduced to proving that

$$
\bigcup_{i \in J} \beta_{i} \subseteq S \cap B(x, C r)
$$


Of course the $\beta_{i}$ 's are all contained in $S$, by their definition, and so it is just a question of showing that $\beta_{i} \subseteq B(x, C r)$ for all $i \in J$. If $i \in J$, then $\operatorname{dist}\left(Q_{i}, S\right) \leq \operatorname{dist}\left(Q_{i}, x\right) \leq r$. Hence $\operatorname{diam} Q_{i} \leq r$, by $(7.1)$, and so $\left|p_{i}-x\right| \leq C r$, by (7.3) and (7.2). We also get that the radius of $\beta_{i}$ is bounded by $C r$, by (7.5) and (7.2). Therefore $\beta_{i} \subseteq B(x, C r)$, and (7.22) follows. Of course (7.19) follows from (7.21) and (7.22), and so the proof of Lemma 7.18 is complete.

Lemma 7.23. $\nu$ is a doubling measure on $\mathbb{R}^{n}$.

Let $x \in \mathbb{R}^{n}$ and $r>0$ be given. We want to prove that

$$
\nu(B(x, 2 r)) \leq C \nu(B(x, r)) .
$$

If $x \in S$, then this follows from Lemma 7.18 and the doubling condition for $\mu$. Thus we may assume that $x \in \mathbb{R}^{n} \backslash S$.

Suppose that $r \geq 2 \operatorname{dist}(x, S)$. Pick a point $z \in S$ such that $\mid x-$ $z \mid=\operatorname{dist}(x, S)$. Then

$$
\nu(B(x, r)) \geq \nu(B(z, r / 2)) .
$$

Since $z \in S$ we can use the preceding case to conclude that

$$
\nu(B(z, r / 2)) \geq C^{-1} \nu(B(z, 3 r)) .
$$

Clearly $B(z, 3 r) \supseteq B(x, 2 r)$, and so we get (7.24) in this case.

Now suppose that $r \leq 10^{-3}$ dist $(x, S)$. Fix a Whitney cube $Q_{i}$ such that $x \in Q_{i}$. Then $\operatorname{dist}(x, S) \leq 6 \operatorname{diam} Q_{i}$, by Lemma 7.8, and therefore every element $z$ of $B(x, 2 r)$ satisfies $\operatorname{dist}\left(z, Q_{i}\right) \leq 2 r \leq \operatorname{diam}\left(Q_{i}\right) / 50$. This means that if $j \in I$ and $Q_{j}$ intersects $B(x, 2 r)$, then $C^{-1} \mu\left(\beta_{i}\right) \leq$ $\mu\left(\beta_{j}\right) \leq C \mu\left(\beta_{i}\right)$, by Lemma 7.12. For this set of $j$ 's -let us call it $J-$ we also have that $\left|Q_{j}\right|$ is comparable to $\left|Q_{i}\right|$, because of Lemma 7.10. Of course $B(x, 2 r)$ does not intersect $S$ in this case, and so we get

$$
\begin{aligned}
\nu(B(x, 2 r)) & =\sum_{j \in J} \frac{\mu\left(\beta_{j}\right)}{\left|Q_{j}\right|}\left|B(x, 2 r) \cap Q_{j}\right| \\
& \leq C \sum_{j \in J} \frac{\mu\left(\beta_{i}\right)}{\left|Q_{i}\right|}\left|B(x, 2 r) \cap Q_{j}\right| \\
& =C \frac{\mu\left(\beta_{i}\right)}{\left|Q_{i}\right|}|B(x, 2 r)|
\end{aligned}
$$




$$
\begin{aligned}
& \leq C \frac{\mu\left(\beta_{i}\right)}{\left|Q_{i}\right|}|B(x, r)| \\
& =C \sum_{j \in J} \frac{\mu\left(\beta_{i}\right)}{\left|Q_{i}\right|}\left|B(x, r) \cap Q_{j}\right| \\
& \leq C \sum_{j \in J} \frac{\mu\left(\beta_{j}\right)}{\left|Q_{j}\right|}\left|B(x, r) \cap Q_{j}\right| \\
& =\nu(B(x, r)) .
\end{aligned}
$$

(In brief, $\nu$ is comparable in size to $\mu\left(\beta_{i}\right) /\left|Q_{i}\right|$ times Lebesgue measure on $B(x, 2 r)$. We shall use this again in the proof of Lemma 7.35 below.) Thus we have (7.24) under these circumstances as well.

We are left with the case where $10^{-3} \operatorname{dist}(x, S)<r<2$ dist $(x, S)$. Again choose $i \in I$ so that $x \in Q_{i}$, and observe that

$$
\nu(B(x, r)) \geq \frac{\mu\left(\beta_{i}\right)}{\left|Q_{i}\right|}\left|B(x, r) \cap Q_{i}\right| \geq C^{-1} \mu\left(\beta_{i}\right) .
$$

This uses Lemma 7.8 too. In this case there is a constant $k>1$ such that

$$
k \beta_{i} \supseteq B(x, 2 r) .
$$

Indeed, the radius of $\beta_{i}$ is comparable to diam $Q_{i}$, and hence to $r$, by Lemma 7.8, and the distance from $x$ to $\beta_{i}$ is bounded by $C \operatorname{diam} Q_{i} \leq$ $C r$, by (7.3), (7.2), and (7.1). The inclusion (7.29) follows from these facts. The doubling condition for $\mu$ together with Lemma 7.18 implies that

$$
\mu\left(\beta_{i}\right) \geq C^{-1} \mu\left(k \beta_{i}\right) \geq C^{-1} \nu\left(k \beta_{i}\right) \geq C^{-1} \nu(B(x, 2 r)) .
$$

Thus we get (7.24) from combining (7.28) and (7.30).

This completes the proof of Lemma 7.23.

REMARK 7.31. For the proof of Lemma 7.23 we did not need to know that $\mu$ is a metric doubling measure on $S$. In other words, if $S$ is a strong set and $\mu$ is doubling on $S$, and if we define $\nu$ as above, then $\nu$ is a doubling measure on $\mathbb{R}^{n}$.

It remains to prove that $\nu$ is a metric doubling measure. Let $\delta_{\mu}(x, y)$ be defined for $x, y \in S$ as in $(1.20)$, and let $\delta_{\nu}(x, y)$ be defined for all $x, y \in \mathbb{R}^{n}$ in the analogous manner. Our assumption that 
$\mu$ be a metric doubling measure on $\mathbb{R}^{n}$ means that there is a metric $d_{\mu}(x, y)$ on $S$ such that

$$
C^{-1} d_{\mu}(x, y) \leq \delta_{\mu}(x, y) \leq C d_{\mu}(x, y)
$$

for all $x, y \in S$.

There is a simple compatibility property between $\delta_{\mu}(x, y)$ and $\delta_{\nu}(x, y)$, which is given by the following.

Lemma 7.33. $\delta_{\mu}(x, y) \leq \delta_{\nu}(x, y) \leq C \delta_{\mu}(x, y)$ for all $x, y \in S$.

This is an easy consequence of Lemma 7.18 and the definitions.

We shall prove that $\nu$ is a metric doubling measure using the following criterion.

Lemma 7.34. In order to show that $\nu$ is a metric doubling measure on $\mathbb{R}^{n}$ it suffices to show that there is a constant $C_{0}$ so that

$$
\delta_{\nu}\left(x_{1}, x_{k}\right) \leq C_{0} \sum_{i=1}^{k-1} \delta_{\nu}\left(x_{i}, x_{i+1}\right)
$$

for any finite sequence $\left\{x_{i}\right\}_{i=1}^{k}$ of points in $\mathbb{R}^{n}, k \geq 2$. (Of course $C_{0}$ is not permitted to depend on $k$.)

This is Lemma 3.1 in [S1]. It is proved by taking $d(x, y)$ to be the infimum of $\sum_{i=1}^{k-1} \delta_{\nu}\left(x_{i}, x_{i+1}\right)$ over all finite sequence $\left\{x_{i}\right\}_{i=1}^{k}$ of points in $\mathbb{R}^{n}, k \geq 2$, which connect $x$ to $y$. The inequality (1.21) follows then from $(7.35)$, and $d(x, y)$ is a metric because the triangle inequality is built into its definition.

Note that the sufficient condition of the lemma is also necessary.

The proof that $\nu$ satisfies this criterion is not difficult but neither is it so lovely. We begin with some minor technical observations.

Lemma 7.36. $\delta_{\nu}(x, y)$ is a quasimetric, i.e., there is a constant $C>0$ so that

$$
\delta_{\nu}(x, z) \leq C\left(\delta_{\nu}(x, y)+\delta_{\nu}(y, z)\right),
$$

for all $x, y, z \in \mathbb{R}^{n}$. 
This is a straightforward consequence of the doubling property for $\nu$.

Lemma 7.38. Define $B(p)$ for $p \in \mathbb{R}^{n}$ by $B(p)=\bar{B}\left(p, 10^{-4} \operatorname{dist}(p, S)\right)$. (Thus $B(p)=\{p\}$ when $p \in S$.) Then there is a number $\lambda=\lambda(p)$ and a constant $C$ such that

$$
C^{-1} \lambda|x-y| \leq \delta_{\nu}(x, y) \leq C \lambda|x-y|
$$

for all $x, y \in B(p)$.

Indeed, let $p$ be given as above, and assume that $p \notin S$, since otherwise the lemma is trivial. Choose $i \in I$ so that $p \in Q_{i}$. The same sort of argument as used in the paragraph containing (7.27) yields

$$
C^{-1} \frac{\mu\left(\beta_{i}\right)}{\left|Q_{i}\right|}|A| \leq \nu(A) \leq C \frac{\mu\left(\beta_{i}\right)}{\left|Q_{i}\right|}|A|
$$

when $A \subseteq 10 B(p)$. Once we have this we get (7.39) from the definition of $\delta_{\nu}(x, y)$, with $\lambda=\left(\mu\left(\beta_{i}\right) /\left|Q_{i}\right|\right)^{1 / n}$. This proves Lemma 7.38.

In Lemma 7.38 we do not have any control over the number $\lambda$, but we do not care. Once we know that $\delta_{\nu}(x, y)$ is comparable to a multiple of the Euclidean metric on $B(p)$ we have the information that we need. (All we really need to know is that it is comparable to some metric there.)

Let us now start to prove that $\nu$ is a metric doubling measure. Let a finite sequence $\left\{x_{i}\right\}_{i=1}^{k}$ of points in $\mathbb{R}^{n}$ be given, as in Lemma 7.34 , and let us try to prove (7.35).

Lemma 7.41. We can find a subsequence $\left\{y_{i}\right\}_{i=1}^{j}$ of $\left\{x_{i}\right\}_{i=1}^{k}$ (i.e., the $y_{i}$ 's are taken from the $x_{i}$ 's, with no repetitions, and the ordering is preserved) with the following properties:

$$
y_{1}=x_{1}, \quad y_{j}=x_{k},
$$

there exists $1 \leq l \leq j$ such that $y_{i+1} \notin B\left(y_{i}\right)$

when $1 \leq i<l$ and $y_{i} \in B\left(y_{l}\right)$ when $i \geq l$,

$$
\sum_{i=1}^{j-1} \delta_{\nu}\left(y_{i}, y_{i+1}\right) \leq C \sum_{i=1}^{k-1} \delta_{\nu}\left(x_{i}, x_{i+1}\right) .
$$


This is pretty easy to prove. Let us first choose some integers $\alpha(m)$ as follows. Set $\alpha(1)=1$. If $x_{i} \in B\left(x_{1}\right)$ for all $i>1$ then we stop, otherwise we choose $\alpha(2)$ to be the smallest $i>1$ such that $x_{i} \notin B\left(x_{1}\right)$. If $\alpha(1), \ldots, \alpha(m)$ have been chosen already then we proceed as follows. If $x_{i} \in B\left(x_{\alpha(m)}\right)$ for all $i>\alpha(m)$ then we stop. Otherwise we choose $\alpha(m+1)$ to be the smallest integer $i>\alpha(m)$ such that $x_{i} \notin B\left(x_{\alpha(m)}\right)$. Of course we are always restricting ourselves to $i \leq k$ here.

Let $l$ denote the largest value of $m$ for which $\alpha(m)$ is defined. Set $y_{i}=x_{\alpha(i)}$ when $1 \leq i \leq l$ and set $y_{l+i}=x_{\alpha(l)+i}$ for as long as this make sense. More precisely, we do nothing for the second definition if $\alpha(l)=k$, and otherwise we use it for $1 \leq i \leq k-\alpha(l)$. This defines our subsequence $\left\{y_{i}\right\}_{i=1}^{j}$, with $j=l+k-\alpha(l)$.

It is not hard to check that (7.42) holds, by construction. We also have (7.43) automatically from our construction.

Let us check (7.44). It suffices to show that

$$
\delta_{\nu}\left(y_{i}, y_{i+1}\right) \leq C \sum_{m=\alpha(i)}^{\alpha(i+1)-1} \delta_{\nu}\left(x_{m}, x_{m+1}\right)
$$

when $i<l$. Keep in mind that $y_{i}=x_{\alpha(i)}$ and $y_{i+1}=x_{\alpha(i+1)}$ here. We may as well assume that $\alpha(i+1)>\alpha(i)+1$, otherwise (7.45) is trivial. By construction we have that $x_{m} \in B\left(x_{\alpha(i)}\right)$ when $\alpha(i) \leq m<\alpha(m+1)$. From Lemma 7.38 we conclude that

$$
\delta_{\nu}\left(x_{\alpha(i)}, x_{\alpha(i+1)-1}\right) \leq C \sum_{m=\alpha(i)}^{\alpha(i+1)-2} \delta_{\nu}\left(x_{m}, x_{m+1}\right)
$$

That is, Lemma 7.38 permits us to get back to the triangle inequality for the Euclidean metric in this case. On the other hand we have that

$$
\begin{aligned}
\delta_{\nu}\left(x_{\alpha(i)}, x_{\alpha(i+1)}\right) \leq C & \left(\delta_{\nu}\left(x_{\alpha(i)}, x_{\alpha(i+1)-1}\right)\right. \\
& \left.+\delta_{\nu}\left(x_{\alpha(i+1)-1}, x_{\alpha(i+1)}\right)\right)
\end{aligned}
$$

by Lemma 7.36. Combining this with (7.46) yields (7.45). The estimate (7.44) follows from (7.45).

Of course it is very important here that these constants $C$ do not depend on $k$ or $l$ or the $x_{i}$ 's, etc.

This completes the proof of Lemma 7.41. 
We let $\left\{y_{i}\right\}_{i=1}^{j}$ and $l$ be as in Lemma 7.41 from now on. In order to prove (7.35) it suffices to show that

$$
\delta_{\nu}\left(y_{1}, y_{j}\right) \leq C \sum_{i=1}^{j-1} \delta_{\nu}\left(y_{i}, y_{i+1}\right)
$$

This assertion follows from (7.42) and (7.44).

Lemma 7.49. In order to prove (7.48) we may assume that $l>1$, and it suffices to show that

$$
\delta_{\nu}\left(y_{1}, y_{l}\right) \leq C \sum_{i=1}^{l-1} \delta_{\nu}\left(y_{i}, y_{i+1}\right)
$$

The point here is that we have replaced the $j$ in (7.48) with $l$. In particular we may as well assume that $l<j$, otherwise there is nothing to do.

To prove the lemma we observe that

$$
\delta_{\nu}\left(y_{l}, y_{j}\right) \leq C \sum_{i=l}^{j-1} \delta_{\nu}\left(y_{i}, y_{i+1}\right)
$$

Indeed, we have that $y_{i} \in B\left(y_{l}\right)$ when $i \geq l$, because of (7.43), and so (7.51) follows from Lemma 7.38. Once we have (7.51) we see that (7.48) is automatic when $l=1$, and that (7.48) would follow when $l>1$ if we had (7.50), because of Lemma 7.36. This proves Lemma 7.49.

Thus we assume from now on that $l>1$, and we want to prove (7.50).

Choose $z_{i} \in S, 1 \leq i \leq l$, so that

$$
\left|z_{i}-y_{i}\right|=\operatorname{dist}\left(y_{i}, S\right)
$$

for each $i$. Thus $z_{i}=y_{i}$ when $y_{i} \in S$.

Lemma 7.53. $\delta_{\nu}\left(z_{i}, y_{i}\right)+\delta_{\nu}\left(z_{i+1}, y_{i+1}\right) \leq C \delta_{\nu}\left(y_{i}, y_{i+1}\right)$ for $1 \leq i<l$.

Indeed, we know from (7.43) that $y_{i+1} \notin B\left(y_{i}\right)$, whence $\left|y_{i+1}-y_{i}\right| \geq$ $10^{-4} \operatorname{dist}\left(y_{i}, S\right)$. This implies that $\left|y_{i+1}-y_{i}\right| \geq 10^{-5} \operatorname{dist}\left(y_{i+1}, S\right)$, as 
one can check. (If $\left|y_{i+1}-y_{i}\right|<10^{-5} \operatorname{dist}\left(y_{i+1}, S\right)$, then dist $\left(y_{i}, S\right) \leq$ $2 \operatorname{dist}\left(y_{i+1}, S\right)$, etc.) Once we have these inequalities it is not hard to derive Lemma 7.53 from the definition of $\delta_{\nu}(x, y)$ and the doubling property for $\nu$.

Lemma 7.54. $\delta_{\nu}\left(z_{1}, z_{l}\right) \leq C \sum_{i=1}^{l-1} \delta_{\nu}\left(y_{i}, y_{i+1}\right)$.

Indeed, Lemma 7.53 implies that

$$
\sum_{i=1}^{l-1} \delta_{\nu}\left(z_{i}, z_{i+1}\right) \leq C \sum_{i=1}^{l-1} \delta_{\nu}\left(y_{i}, y_{i+1}\right)
$$

Here is where we use our hypothesis that $\mu$ is a metric doubling measure. Because the $z_{i}$ 's lie in $S$ we have that $\delta_{\mu}\left(z_{i}, z_{i+1}\right) \leq \delta_{\nu}\left(z_{i}, z_{i+1}\right)$ for each $i$, as in Lemma 7.33. Thus

$$
\sum_{i=1}^{l-1} \delta_{\mu}\left(z_{i}, z_{i+1}\right) \leq \sum_{i=1}^{l-1} \delta_{\nu}\left(z_{i}, z_{i+1}\right)
$$

The metric doubling condition for $\mu$ (see (7.32)) implies that

$$
\delta_{\mu}\left(z_{1}, z_{l}\right) \leq C \sum_{i=1}^{l-1} \delta_{\mu}\left(z_{i}, z_{i+1}\right) .
$$

Combining these inequalities we get

$$
\delta_{\mu}\left(z_{1}, z_{l}\right) \leq C \sum_{i=1}^{l-1} \delta_{\nu}\left(y_{i}, y_{i+1}\right) .
$$

This implies Lemma 7.54, because of Lemma 7.33.

Lemma 7.59. $\delta_{\nu}\left(y_{1}, y_{l}\right) \leq C \sum_{i=1}^{l-1} \delta_{\nu}\left(y_{i}, y_{i+1}\right)$.

This follows from Lemmas 7.54, 7.53, and 7.36.

Lemma 7.59 asserts the validity of (7.50). Lemma 7.49 implies that (7.48) holds, and we saw already that (7.48) implies that (7.35) is true. 
Thus we have proved that $\nu$ satisfies the criterion for being a metric doubling measure in Lemma 7.34.

This completes the proof of Proposition 1.23.

Acknowledgements. I am grateful to Guy David, Juha Heinonen, Jussi Väisälä, and the referee for some helpful discussions about this matter.

\section{References.}

[BA] Beurling, A. and Ahlfors, L., The boundary correspondence under quasiconformal mappings. Acta Math. 96 (1956), 125-142.

[DS] David, G., Semmes, S., Strong $A_{\infty}$-weights, Sobolev inequalities, and quasiconformal mappings in Analysis and Partial Differential Equations. Edited by C. Sadosky, Lecture Notes in Pure and Applied Mathematics 122, Marcel Dekker 1990.

[G] Gehring, F. W., The $L^{p}$ integrability of the partial derivatives of a quasiconformal mapping. Acta Math. 130 (1973), 265-277.

[S1] Semmes, S., Bilipschitz mappings and strong $A_{\infty}$-weights. Ann. Acad. Sci. Fenn. Series A I Math. 18 (1993), 211-248.

[S2] Semmes, S., On the nonexistence of bilipschitz parameterizations and geometric problems about $A_{\infty}$-weights. Revista Mat. Iberoamericana 12 (1996), 337-410.

[St] Stein, E. M., Singular Integrals and Differentiability Properties of Functions. Princeton Univ. Press, 1970.

[TV] Tukia, P., Väisälä, J., Quasisymmetric embeddings of metric spaces. Ann. Acad. Sci. Fenn. Series A I Math. 5 (1980), 97-114.

[V1] Väisälä, J., Lectures on n-dimensional quasiconformal mappings. Lecture Notes in Math. 229, Springer-Verlag, 1971.

[V2] Väisälä, J., Quasi-symmetric embeddings in Euclidean spaces. Trans. Amer. Math. Soc. 264 (1981), 191-204.

[V3] Väisälä, J., Porous sets and quasisymmetric maps. Trans. Amer. Math. Soc. 299 (1987), 525-533. 
[VVW] Väisälä, J., Vuorinen, M., Wallin, H., Thick sets and quasisymmetric maps. Nagoya Math. J. 135 (1994), 121-148.

Recibido: 6 de octubre de 1.995

Revisado: 10 de noviembre de 1.995

\author{
Stephen Semmes* \\ Department of Mathematics \\ Rice University \\ Houston, Texas 77251, U.S.A. \\ semmes @math . rice.edu \\ and \\ I.H.E.S. \\ 35 Route de Chartres \\ 91440 Bures-sur-Yvette, FRANCE \\ semmes@ihes.fr
}

\footnotetext{
* The author was supported in part by the U.S. National Science Foundation, and is grateful to IHES for its hospitality.
} 